\title{
Splitting Numbers of Grids
}

\author{
Dwight Duffus \\ Mathematics and Computer Science Department \\ Emory University \\ Atlanta GA 30322 USA \\ dwight@mathcs . emory.edu \\ Bill Sands \\ Mathematics and Statistics Department \\ The University of Calgary \\ Calgary AB T2N 1N4 CANADA \\ sands@math . ucalgary . ca
}

Submitted: Nov 10, 2003; Accepted: Apr 4, 2005; Published: Apr 13, 2005

MR Subject Classifications [2000]: 06A07, 06D99

\begin{abstract}
For a subset $S$ of a finite ordered set $P$, let

$S \uparrow=\{x \in P: x \geq s$ for some $s \in S\} \quad$ and $\quad S \downarrow=\{x \in P: x \leq s$ for some $s \in S\}$.

For a maximal antichain $A$ of $P$, let

$$
s(A)=\max _{A=U \cup D} \frac{|U \uparrow|+|D \downarrow|}{|P|},
$$

the maximum taken over all partitions $U \cup D$ of $A$, and

$$
s_{k}(P)=\min _{A \in \mathcal{A}(P),|A|=k} s(A)
$$

where we assume $P$ contains at least one maximal antichain of $k$ elements. Finally, for a class $\mathcal{C}$ of finite ordered sets, we define

$$
s_{k}(\mathcal{C})=\inf _{P \in \mathcal{C}} s_{k}(P)
$$

Thus $s_{k}(\mathcal{C})$ is the greatest proportion $r$ satisfying: every $k$-element maximal antichain of a member $P$ of $\mathcal{C}$ can be "split" into sets $U$ and $D$ so that $U \uparrow \cup D \downarrow$ contains at least $r|P|$ elements.

In this paper we determine $s_{k}\left(\mathcal{G}_{k}\right)$ for all $k \geq 1$, where $\mathcal{G}_{k}=\{\mathbf{k} \times \mathbf{n}: n \geq k\}$ is the family of all $k$ by $n$ "grids".
\end{abstract}




\section{Introduction}

Given a maximal antichain $A$ of an ordered set $P$, say that $A$ splits if there is a partition $A=U \cup D$ such that $P=U \uparrow \cup D \downarrow$, where

$$
U \uparrow=\{x \in P: x \geq u \text { for some } u \in U\} \quad \text { and } \quad D \downarrow=\{x \in P: x \leq d \text { for some } d \in D\} \text {. }
$$

Say that $P$ has the splitting property if every maximal antichain of $P$ splits. Ahlswede, Erdős and Graham introduced these notions in [1], and proved that every finite Boolean lattice has the splitting property. In [2] we used the splitting property to study maximal antichains in distributive lattices. More recently, in [3], we characterized the set of distributive lattices with the splitting property, and also introduced the idea of a splitting number for any finite ordered set and any class of finite ordered sets. We restate the required definitions.

For a maximal antichain $A$ of a finite ordered set $P$, let

$$
s(A)=\max _{A=U \cup D} \frac{|U \uparrow|+|D \downarrow|}{|P|},
$$

the maximum taken over all partitions $U \cup D$ of $A$. Define the splitting number of $P$ to be

$$
s(P)=\min _{A \in \mathcal{A}(P)} s(A),
$$

where $\mathcal{A}(P)$ is the set of all maximal antichains of $P$. Furthermore, if $\mathcal{C}$ is a class of finite ordered sets, we define the splitting number of $\mathcal{C}$ to be

$$
s(\mathcal{C})=\inf _{P \in \mathcal{C}} s(P)
$$

We also make analogous definitions when the antichains involved are restricted to a certain size: for a finite ordered set $P$, or a class $\mathcal{C}$ of finite ordered sets, let

$$
s_{k}(P)=\min _{A \in \mathcal{A}(P),|A|=k} s(A) \quad \text { and } \quad s_{k}(\mathcal{C})=\inf _{P \in \mathcal{C}} s_{k}(P)
$$

where $k$ is a positive integer such that $P$ contains at least one maximal antichain of $k$ elements. Thus $s_{k}(\mathcal{C})$ is the greatest proportion $r$ satisfying: every $k$-element maximal antichain of a member $P$ of $\mathcal{C}$ can be "split" into sets $U$ and $D$ so that $U \uparrow \cup D \downarrow$ contains at least $r|P|$ elements. The same condition with the restriction on antichain size removed yields $s(\mathcal{C})$. It is clear that $s(P) \leq s_{k}(P)$ and $s(\mathcal{C}) \leq s_{k}(\mathcal{C})$ for all $k$.

Note that the Ahlswede-Erdős-Graham theorem [1] could be stated as: $s(\mathcal{B})=1$ where $\mathcal{B}$ is the class of all finite Boolean lattices. Also, it's not difficult to see that if $\mathcal{P}$ is the class of all finite ordered sets, $s(\mathcal{P})=s_{k}(\mathcal{P})=1 / 2$ for all $k$. The problem of determining $s(\mathcal{C})$ and $s_{k}(\mathcal{C})$ for various classes is an interesting order-theoretic and combinatorial task. When $\mathcal{C}$ is the family of all finite distributive lattices, for instance, we only have bounds (and not very good ones) in [3]. But the more restricted family $\mathcal{G}_{k}=\{\mathbf{k} \times \mathbf{n}: n \geq k\}$ of all $k$ by $n$ "grids", where $k$ is fixed and $n \geq k$, appeared to 
us to present a challenging but attainable goal, and in [3] we began to determine $s_{k}\left(\mathcal{G}_{k}\right)$. It is not difficult to show that $\lim _{k \rightarrow \infty} s_{k}\left(\mathcal{G}_{k}\right)=1$, and at the time we had a guess for what $s_{k}\left(\mathcal{G}_{k}\right)$ was, linked closely to the Pell numbers and a "Pascal-like" triangle. Here, we present verification of our guess.

Theorem 1 For all positive integers $k$,

$$
s_{k}\left(\mathcal{G}_{k}\right)=1-\frac{1}{k}+\frac{1}{k y_{k}}
$$

where $y_{k}$ is defined by: $y_{1}=2, y_{2}=3, y_{3}=6$, and

$$
y_{k}= \begin{cases}2 y_{k-1}-y_{k-4} & \text { for } k \text { odd }, \\ 2 y_{k-1}-y_{k-2} & \text { for } k \text { even } .\end{cases}
$$

Thus the sequence $y_{1}, y_{2}, \ldots$ starts $2,3,6,9,16,23,40,57,98,139,238, \ldots$ and we get

$$
s_{1}\left(\mathcal{G}_{1}\right)=\frac{1}{2}, \quad s_{2}\left(\mathcal{G}_{2}\right)=\frac{2}{3}, \quad s_{3}\left(\mathcal{G}_{3}\right)=\frac{13}{18}, \quad s_{4}\left(\mathcal{G}_{4}\right)=\frac{7}{9},
$$

and so on, as reported in [3]. The first two values are derived from general results for distributive lattices. The values for $k=3$ through $k=6$ were obtained with an early version of the strategy fully developed in this paper.

Actually, the result we obtain is stronger: for each odd $k$ there is, in a certain sense, a "unique" antichain which realizes the minimum splitting value. This is made precise in Theorem 2 in Section 5. For even $k$, there does not appear to be uniqueness, unless perhaps symmetry is imposed.

Here is an outline of the paper. Section 2 contains definitions and notation. We employ matrix notation in the proof of Theorem 1 and the required material is provided in Sections 2 and 3. Section 3 also contains our proof that the value given in Theorem 1 is a lower bound for $s_{k}\left(\mathcal{G}_{k}\right)$. The converse inequality is verified in Section 4 . In Section 5 is the promised description and proof of uniqueness. Finally in Section 6 we show that for even $k$ we cannot obtain the same uniqueness result and state some open problems.

\section{Preliminaries}

We represent a $k$-element maximal antichains in the lattice $L=\mathbf{k} \times \mathbf{n}$ as a vector of nonnegative integers. Assume that the chains are labelled so that $\mathbf{k}=\{1<2<\ldots<k\}$ and $\mathbf{n}=\{1<2<\ldots<n\}$. Given a $k$-element maximal antichain $A=\left\{a_{1}, a_{2}, \ldots, a_{k}\right\}$, we can put $a_{1}=\left(k, n_{1}\right), a_{2}=\left(k-1, n_{1}+n_{2}\right)$, and in general $a_{i}=\left(k+1-i, n_{1}+n_{2}+\cdots+n_{i}\right)$, where $n_{i} \geq 1$ for all $1 \leq i \leq k$ and $\sum_{i=1}^{k} n_{i} \leq n$. Letting $n_{k+1}=n-\sum_{i=1}^{k} n_{i} \geq 0$, we have a representation of $A$ by the vector $\bar{n}=\left(n_{1}, n_{2}, \ldots, n_{k+1}\right)$. It is clear that this provides a 1-1 correspondence between $k$-antichains of $L$ and $(k+1)$-vectors of integers

$\bar{n}=\left(n_{1}, n_{2}, \ldots, n_{k+1}\right)$ where $\sum_{i=1}^{k+1} n_{i}=n, n_{i}>0(i=1,2, \ldots, k)$ and $n_{k+1} \geq 0$. 
Given a maximal antichain $A$ of $L$, there is a corresponding natural partition

$$
\{N(i, j): 1 \leq i \leq k, 1 \leq j \leq k+1\}
$$

of $L$ into intervals $N(i, j)$, where

$$
N(i, j)=\left\{(i, v) \in L: \sum_{t=1}^{j-1} n_{t}<v \leq \sum_{t=1}^{j} n_{t}\right\} \quad \text { for } \quad 1 \leq i \leq k \quad \text { and } \quad 1 \leq j \leq k+1 .
$$

For all $i, j,|N(i, j)|=n_{j}$. (See Figure 1.)

An orientation $o$ of a maximal antichain $A$ is an ordered pair $(U, D)$ where $A$ is partitioned by $U$ and $D$. We say that o captures the elements in $U \uparrow \cup D \downarrow$. We assign $\uparrow$ 's to the elements of $U$ and $\downarrow$ 's to those of $D$. For instance, if $o$ has $U=\left\{a_{1}, a_{3}, a_{5}, \ldots\right\}$ and $D=\left\{a_{2}, a_{4}, a_{6}, \ldots\right\}$, we denote $o$ by $a_{1} \uparrow a_{2} \downarrow a_{3} \uparrow a_{4} \downarrow \ldots$. With the elements of $A$ in their natural order, the $a_{i}$ 's can be dropped and an orientation can be defined by a $k$-sequence of $\uparrow$ 's and $\downarrow$ 's — the "alternating" orientation above is just $\uparrow \downarrow \uparrow \downarrow \uparrow \downarrow \ldots$.

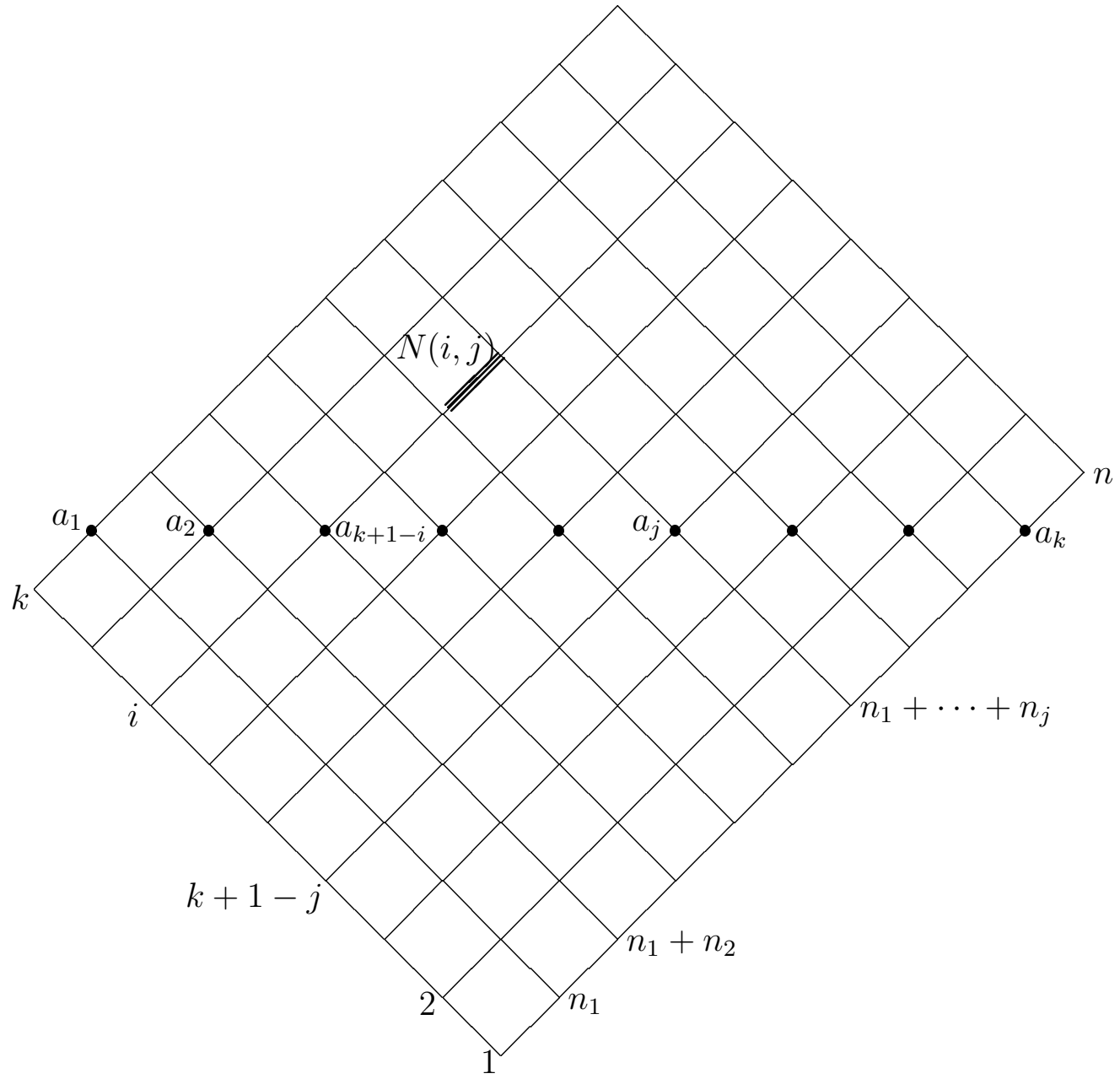

Figure 1: an interval $N(i, j)$ 
The reverse of an orientation $o$ is the orientation $o^{r}$ obtained by both reversing the order of the arrows and replacing each $\uparrow$ by a $\downarrow$ and vice versa; so for $o=\uparrow \uparrow \downarrow$ we would get $o^{r}=\uparrow \downarrow \downarrow$ for example. (See Figure 2.) An orientation is self-reversing if it is equal to its reverse.
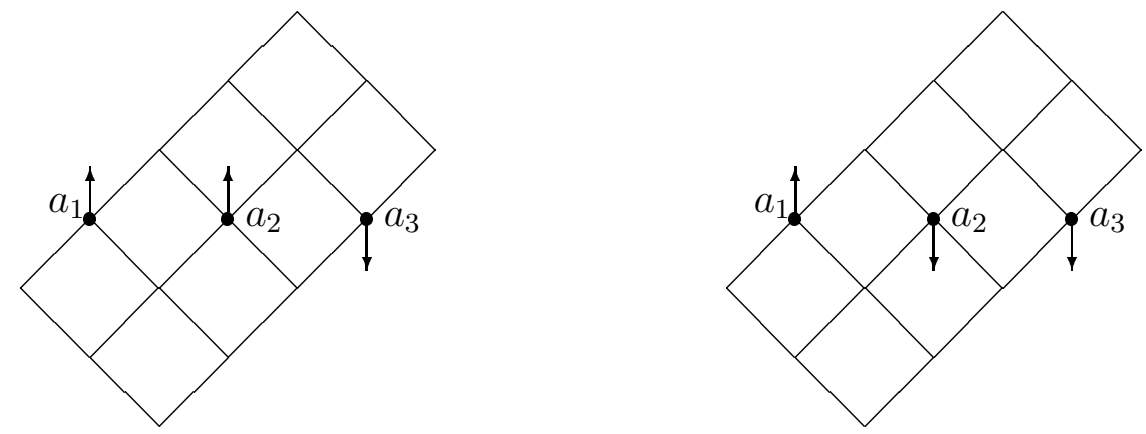

Figure 2: an orientation and its reverse

Most of the time, if any element of $N(i, j)$ is captured by an orientation $o$ then all are. The only exception is that $o$ can capture the greatest element $\left(i, n_{1}+\cdots+n_{j}\right)$ of $N(i, j)$ without capturing all of $N(i, j)$. This happens for such an $N(i, j)$ precisely if $i+j \geq k+1$, $a_{j} \uparrow$, and $a_{r} \downarrow$ for all $r$ such that $k+1-i \leq r<j$; in this case all elements $\left(r, \sum_{l=1}^{j} n_{l}\right)$ for $k+1-j \leq r \leq i$ are exceptional. In fact, $o$ captures $r_{1} n_{1}+r_{2} n_{2}+\cdots+r_{k+1} n_{k+1}+r_{0}$ elements of $L$, where $r_{j}(j \geq 1)$ is the number of indices $i$ such that $N(i, j)$ is captured by $o$, and $r_{0}$ is the number of exceptional elements $\left(r, n_{1}+\cdots+n_{j}\right)$ captured by $o$ but not in an interval captured by $o$. Note that $0 \leq r_{0} \leq k$, since there is at most one such exceptional element with a given first coordinate. Define the capture vector $\bar{v}_{o}$ induced by $o$ to be the $(k+1)$-vector $\left(r_{1}, r_{2}, \ldots, r_{k+1}\right)$. Then the number of elements captured by $o$ is $\bar{v}_{o} \cdot \bar{n}+r_{0}$, the dot denoting the dot product of the vectors.

Here is a simple result which we will need later. The reverse $\bar{v}^{r}$ of a vector $\bar{v}$ is obtained by writing the components of $\bar{v}$ in reverse order.

Lemma 1 For any orientation o,

$$
\bar{v}_{o^{r}}=\left(\bar{v}_{o}\right)^{r} .
$$

Finally, the methods we develop to prove Theorem 1 do not apply in case $k=2$. As noted above, for small values of $k$, the result in Theorem 1 was obtained in [3]. Where needed, we are free to assume $k \neq 2$ in Sections 3 and 4.

\section{The lower bound}

Let

$$
s_{k}=1-\frac{1}{k}+\frac{1}{k y_{k}}
$$


be the quantity given in Theorem 1 . Our goal here is to prove that every maximal $k$ antichain of a lattice $L=\mathbf{k} \times \mathbf{n} \in \mathcal{G}_{k}$ can be oriented so as to capture at least $s_{k}|L|$ elements of $L$. This will prove that $s_{k}\left(\mathcal{G}_{k}\right) \geq s_{k}$.

To establish the lower bound, we show that for any antichain $A$ with associated vector $\bar{n}$ as defined above, there is an orientation $o$ such that $\bar{v}_{o} \cdot \bar{n} \geq s_{k}|L|=s_{k} k n$. Our method is to find a nonempty set $\mathcal{O}=\left\{o_{1}, \ldots, o_{m}\right\}$ of orientations, and positive numbers $\lambda_{i}$, $1 \leq i \leq m$, so that

$$
\sum_{i=1}^{m} \lambda_{i}\left(\bar{v}_{o_{i}} \cdot \bar{n}\right)=s_{k} k n \sum_{i=1}^{m} \lambda_{i}
$$

for all $\bar{n}$. It follows that at least one of the $o_{i}$ 's in $\mathcal{O}$ satisfies $\bar{v}_{o_{i}} \cdot \bar{n} \geq s_{k} k n$, so $s_{k}\left(\mathcal{G}_{k}\right) \geq s_{k}$ as desired. It turns out we can select $\mathcal{O}$ and the $\lambda_{i}$ 's independently of $\bar{n}$.

Arranging the capture vectors of the $m$ orientations in the set $\mathcal{O}$ as rows of a matrix, we obtain the $m$ by $k+1$ capture matrix $M_{k}$ of $\mathcal{O}$. Now we can rewrite (1) in matrix form as

$$
\bar{n} M_{k}^{t} \bar{\lambda}=\bar{n} s_{k} k J \bar{\lambda}
$$

where $\bar{n}$ is a 1 -by- $(k+1)$ row vector, $\bar{\lambda}=\left(\lambda_{1}, \ldots, \lambda_{m}\right)$ is an $m$-by-1 column vector, and $J$ is a matrix of 1 's of appropriate size, in this case $(k+1)$-by- $m$. This equation can be written as

$$
\bar{n}\left(M_{k}^{t}-s_{k} k J\right) \bar{\lambda}=0,
$$

so it certainly suffices to prove that

$$
\left(M_{k}^{t}-s_{k} k J\right) \bar{\lambda}=\overline{0}
$$

where $\overline{0}$ is a zero column vector of length $m$.

Now we will define the set $\mathcal{O}$ of $m$ orientations and the associated $m$-vector $\bar{\lambda}$. It turns out that we can let $m=k+1$.

Define the orientations

$$
a_{\uparrow}:\left\{\begin{array}{ll}
\uparrow \downarrow \uparrow \downarrow \uparrow \downarrow \ldots \uparrow & \text { for } k \text { odd, } \\
\uparrow \downarrow \uparrow \downarrow \uparrow \downarrow \ldots \downarrow & \text { for } k \text { even, }
\end{array} \quad a_{\downarrow}:\left\{\begin{array}{lll}
\downarrow \uparrow \downarrow \uparrow \downarrow \ldots & \text { for } k \text { odd, } \\
\downarrow \uparrow \downarrow \uparrow \downarrow \ldots \uparrow & \text { for } k \text { even, }
\end{array}\right.\right.
$$

and

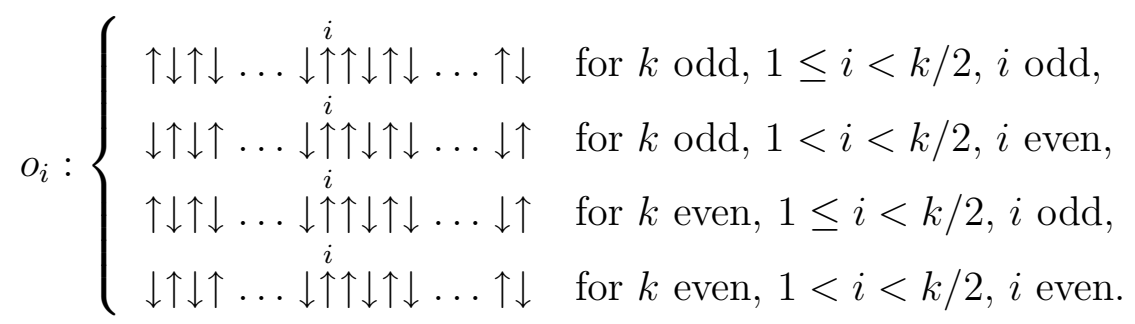

Also, for $k$ even and at least 4 , define the orientation

$$
O O: \uparrow \uparrow \downarrow \uparrow \downarrow \uparrow \ldots \downarrow \uparrow \downarrow \downarrow \text {. }
$$

The capture vectors of these orientations are given in the following tables, the first is for $k$ odd, and the second is for $k$ even. All vectors, including the constant vector $\overline{k-1}=(k-1, k-1, \ldots, k-1)$, have length $k+1$. 


\begin{tabular}{|c|c|}
\hline orientation $o$ & capture vector $\bar{v}_{o}$ \\
\hline$a_{\uparrow}$ & $\overline{k-1}+(0,1,-1,1,-1, \ldots,-1,1)$ \\
$a_{\downarrow}$ & $\frac{\bar{k}-1}{k-1}+(-1,-1,1,1,-1, \ldots,-1,-1,1, \ldots,-1,1,0)$ \\
$o_{1}$ & $i=1,0)$ \\
$o_{i}, 1<i<k / 2, i$ odd & $\overline{k-1}+(0,1,-1,1,-1, \ldots,-1,1,-2,0,1,-1,1,-1, \ldots,-1,1,0)$ \\
$o_{i}, 1<i<k / 2, i$ even & $\overline{k-1}+(1,-1,1,-1, \ldots,-1,1,-2,0,1,-1,1,-1, \ldots,-1,1)$, \\
\hline
\end{tabular}

\begin{tabular}{|c|c|}
\hline orientation $o$ & capture vector $\bar{v}_{o}$ \\
\hline$a_{\uparrow}$ & $\overline{k-1}+(0,1,-1,1,-1, \ldots,-1,1,0)$ \\
$a_{\downarrow}$ & $\overline{k-1}+(1,-1,1,-1, \ldots,-1,1)$ \\
$o_{1}$ & $\overline{k-1}+(-1,0,1,-1,1,-1,1, \ldots,-1,1)$ \\
$o_{i}, 1<i<k / 2, i$ odd & $\overline{k-1}+(0,1,-1,1,-1, \ldots,-1,1,-2,0,1,-1,1,-1, \ldots,-1,1)$ \\
$o_{i}, 1<i<k / 2, i$ even & $\overline{k-1}+(1,-1,1,-1, \ldots,-1,1,-2,0,1,-1,1,-1, \ldots,-1,1,0)$, \\
$o$ oo & $\overline{k-1}+(-1,0,1,-1,1,-1, \ldots,-1,1,0,-1)$ \\
\hline
\end{tabular}

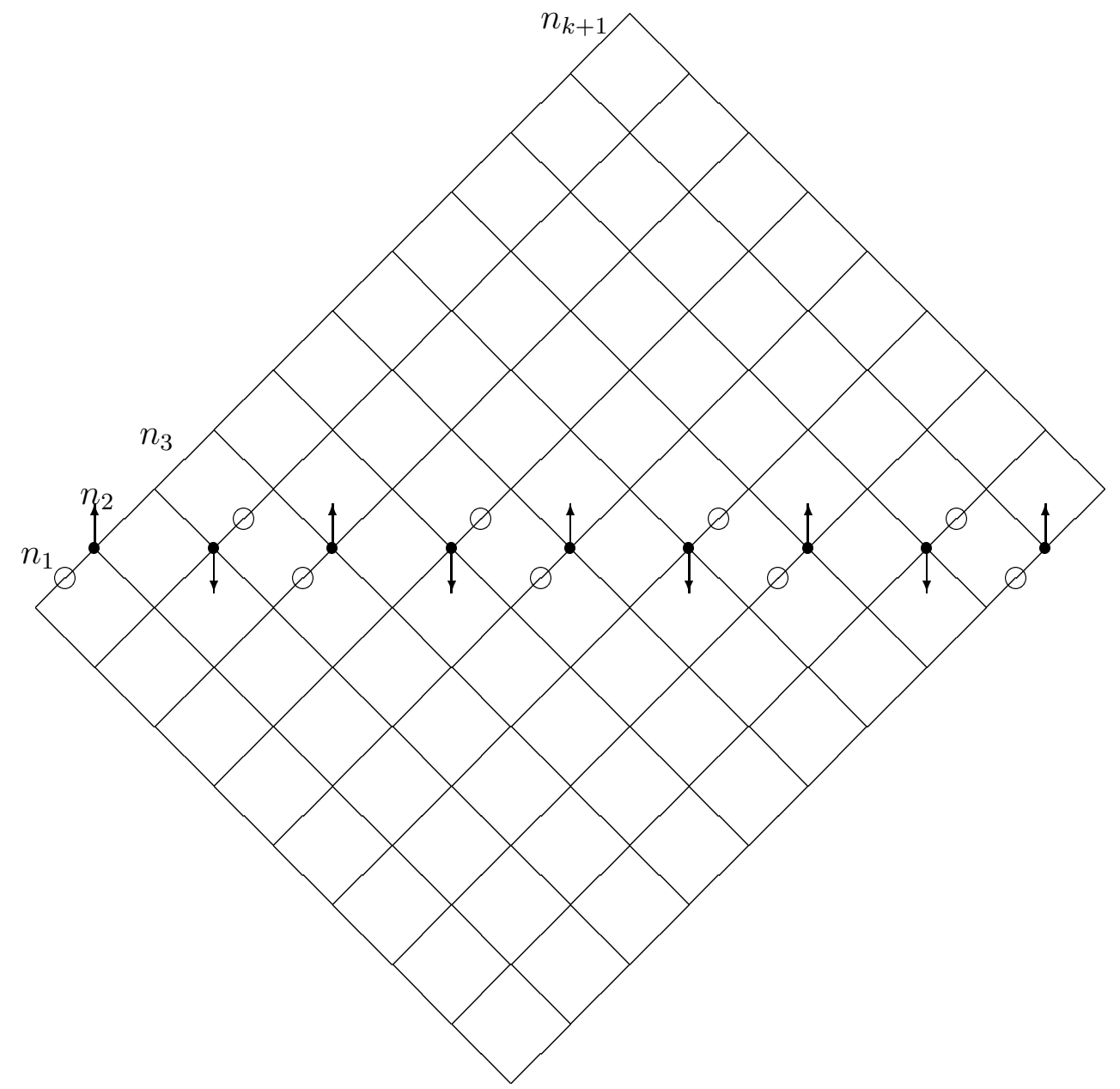

Figure 3: finding the capture vector of $a_{\uparrow}$ 
Figure 3 shows the alternating orientation $a_{\uparrow}$ applied to a maximal antichain of an arbitrary lattice in $\mathcal{G}_{k}$ for odd $k$. The antichain elements are shown as small solid circles, and larger hollow circles show intervals which are not captured by the orientation. One sees that exactly $k-1$ intervals (out of $k$ ) of length $n_{1}$ are captured, all $k$ of the length $n_{2}$ intervals are captured, $k-2$ of the length $n_{3}$ intervals are captured, and so on, giving

$$
\bar{v}_{a_{\uparrow}}=(k-1, k, k-2, k, k-2, \ldots, k, k-2, k)=\overline{k-1}+(0,1,-1,1,-1, \ldots,-1,1)
$$

as claimed. The other capture vectors can be similarly checked.

Note that $a_{\downarrow}$ is the reverse of $a_{\uparrow}$ when $k$ is odd, but not when $k$ is even. When $k$ is even, the orientations $a_{\uparrow}, a_{\downarrow}$ and oo are all self-reversing.

For $k$ odd, we let

$$
\mathcal{O}=\left\{a_{\uparrow}, o_{(k-1) / 2}, o_{(k-3) / 2}, \ldots, o_{2}, o_{1}, o_{1}^{r}, o_{2}^{r}, \ldots, o_{(k-1) / 2}^{r}, a_{\downarrow}\right\} .
$$

Note that $|\mathcal{O}|=k+1$. As noted in Lemma 1, the capture vector of $o^{r}$ is the reverse of the capture vector of $o$. Thus with the orientations of $\mathcal{O}$ ordered as listed, we obtain the $(k+1) \times(k+1)$ capture matrix

$$
M_{k}=(k-1) J_{k+1}+C_{k},
$$

where $J_{k+1}$ is the square all-ones matrix of order $k+1$,

$$
C_{k}=\left(\begin{array}{ccccccccccc|ccccccccc}
0 & 1 & -1 & 1 & -1 & \ldots & -1 & 1 & -1 & 1 & -1 & 1 & -1 & 1 & -1 & \ldots & -1 & 1 & -1 & 1 \\
1 & -1 & 1 & -1 & 1 & \ldots & 1 & -1 & 1 & -2 & 0 & 1 & -1 & 1 & -1 & \ldots & -1 & 1 & -1 & 1 \\
0 & 1 & -1 & 1 & -1 & \ldots & -1 & 1 & -2 & 0 & 1 & -1 & 1 & -1 & 1 & \ldots & 1 & -1 & 1 & 0 \\
1 & -1 & 1 & -1 & 1 & \ldots & 1 & -2 & 0 & 1 & -1 & 1 & -1 & 1 & -1 & \ldots & -1 & 1 & -1 & 1 \\
& & & & & \vdots & & & & & & & & & & \vdots & & & \\
\\
0 & 1 & -2 & 0 & 1 & \ldots & 1 & -1 & 1 & -1 & 1 & -1 & 1 & -1 & 1 & \ldots & 1 & -1 & 1 & 0 \\
1 & -2 & 0 & 1 & -1 & \ldots & -1 & 1 & -1 & 1 & -1 & 1 & -1 & 1 & -1 & \ldots & -1 & 1 & -1 & 1 \\
-1 & 0 & 1 & -1 & 1 & \ldots & 1 & -1 & 1 & -1 & 1 & -1 & 1 & -1 & 1 & \ldots & 1 & -1 & 1 & 0 \\
\hline & & & & & & & & & & & & & & & & & & & \\
\\
0 & 1 & -1 & 1 & -1 & \ldots & -1 & 1 & -1 & 1 & -1 & 1 & -1 & 1 & -1 & \ldots & -1 & 1 & 0 & -1 \\
1 & -1 & 1 & -1 & 1 & \ldots & 1 & -1 & 1 & -1 & 1 & -1 & 1 & -1 & 1 & \ldots & 1 & 0 & -2 & 1 \\
0 & 1 & -1 & 1 & -1 & \ldots & -1 & 1 & -1 & 1 & -1 & 1 & -1 & 1 & -1 & \ldots & 0 & -2 & 1 & 0 \\
1 & -1 & 1 & -1 & 1 & \ldots & 1 & -1 & 1 & -1 & 1 & -1 & 1 & -1 & 1 & \ldots & -2 & 1 & -1 & 1 \\
& & & & & \vdots & & & & & & & & & & \vdots & & & \\
0 & 1 & -1 & 1 & -1 & \ldots & -1 & 1 & -1 & 1 & -1 & 1 & 0 & -2 & 1 & \ldots & 1 & -1 & 1 & 0 \\
1 & -1 & 1 & -1 & 1 & \ldots & 1 & -1 & 1 & -1 & 1 & 0 & -2 & 1 & -1 & \ldots & -1 & 1 & -1 & 1 \\
1 & -1 & 1 & -1 & 1 & \ldots & 1 & -1 & 1 & -1 & 1 & -1 & 1 & -1 & 1 & \ldots & 1 & -1 & 1 & 0
\end{array}\right)
$$

when $k \equiv 1 \bmod 4$, and 


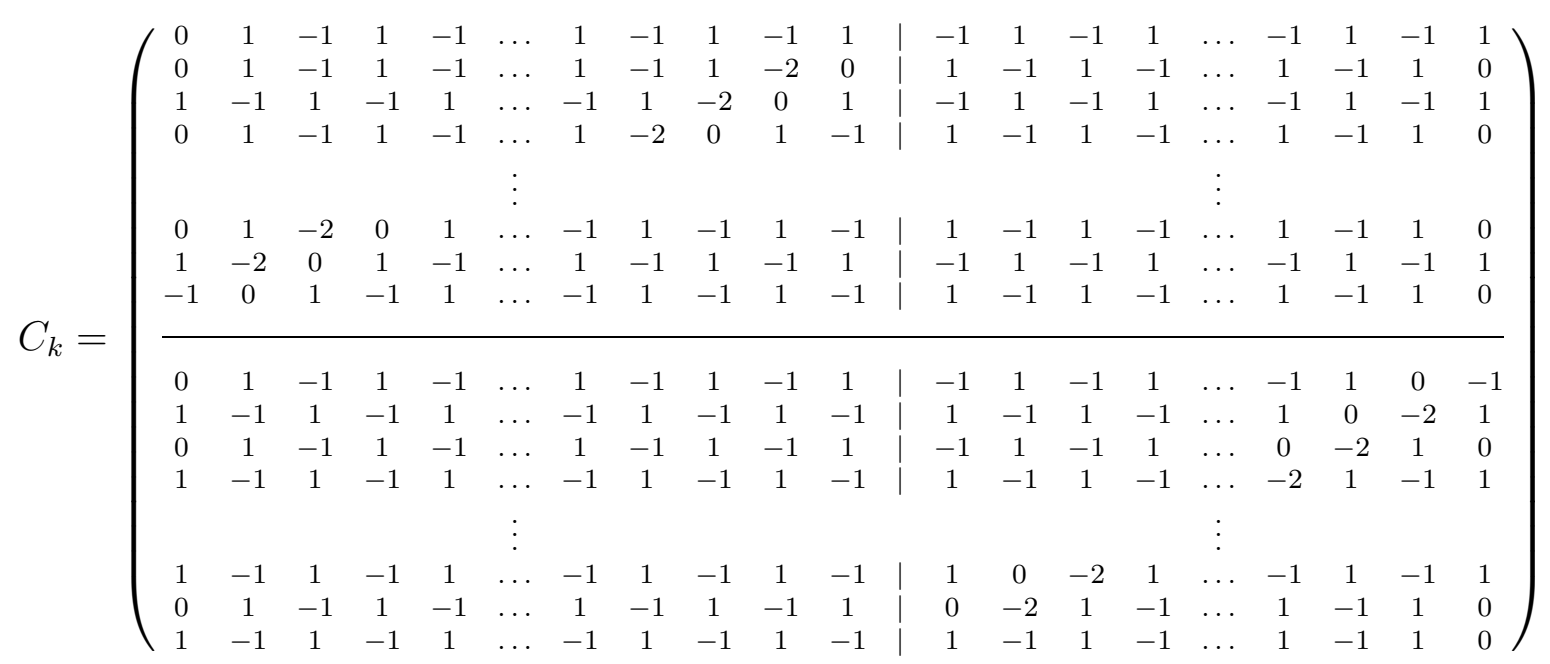

when $k \equiv 3 \bmod 4$. [Note: the horizontal and vertical lines divide $C_{k}$ into four square submatrices of order $(k+1) / 2$.]

For $k$ even and at least 4 , we similarly define

$$
\mathcal{O}=\left\{a_{\uparrow}, o_{(k / 2)-1}, o_{(k / 2)-2}, \ldots, o_{2}, o_{1}, o o, o_{1}^{r}, o_{2}^{r}, \ldots, o_{(k / 2)-1}^{r}, a_{\downarrow}\right\}
$$

Again $|\mathcal{O}|=k+1$, and this time the $(k+1) \times(k+1)$ capture matrix is

$$
M_{k}=(k-1) J_{k+1}+C_{k}
$$

where

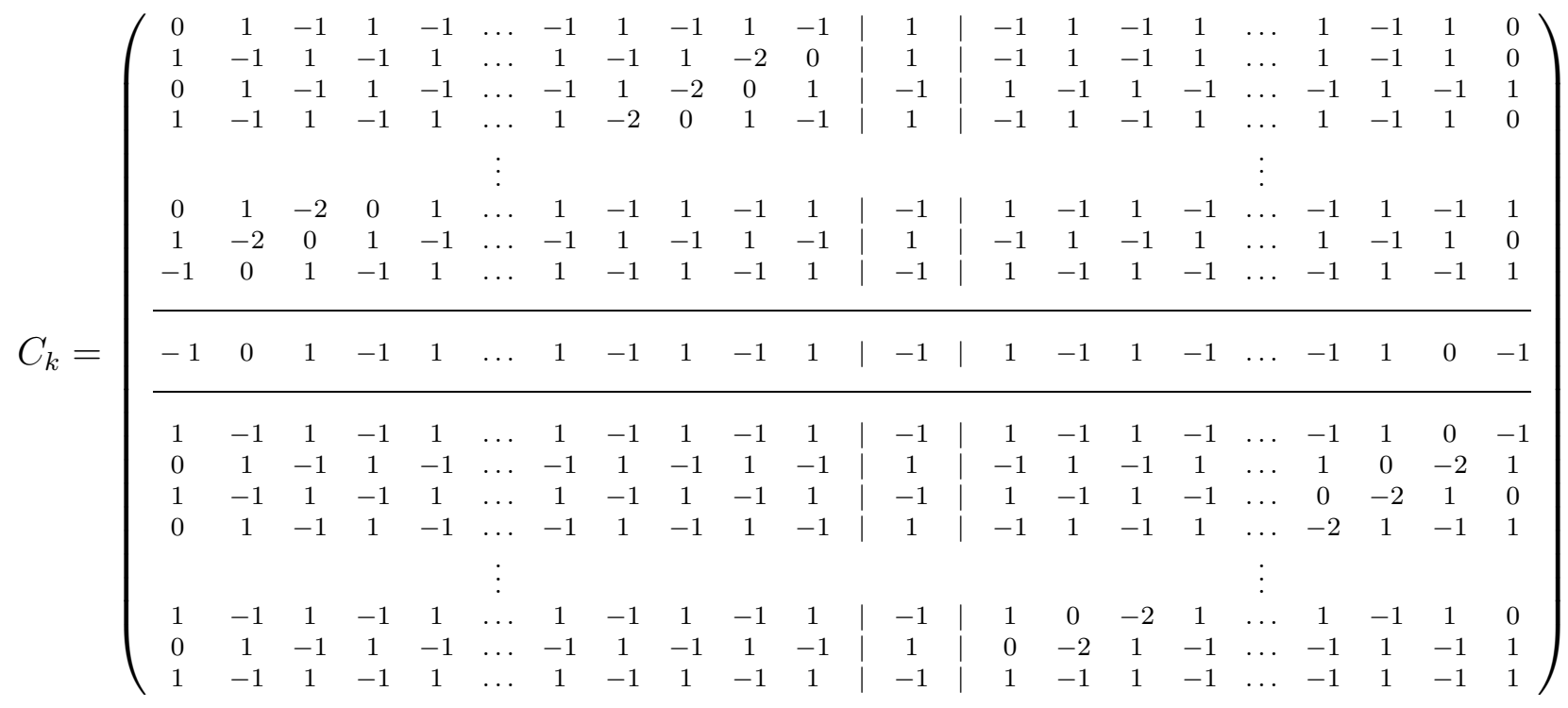

when $k \equiv 2 \bmod 4$, and 


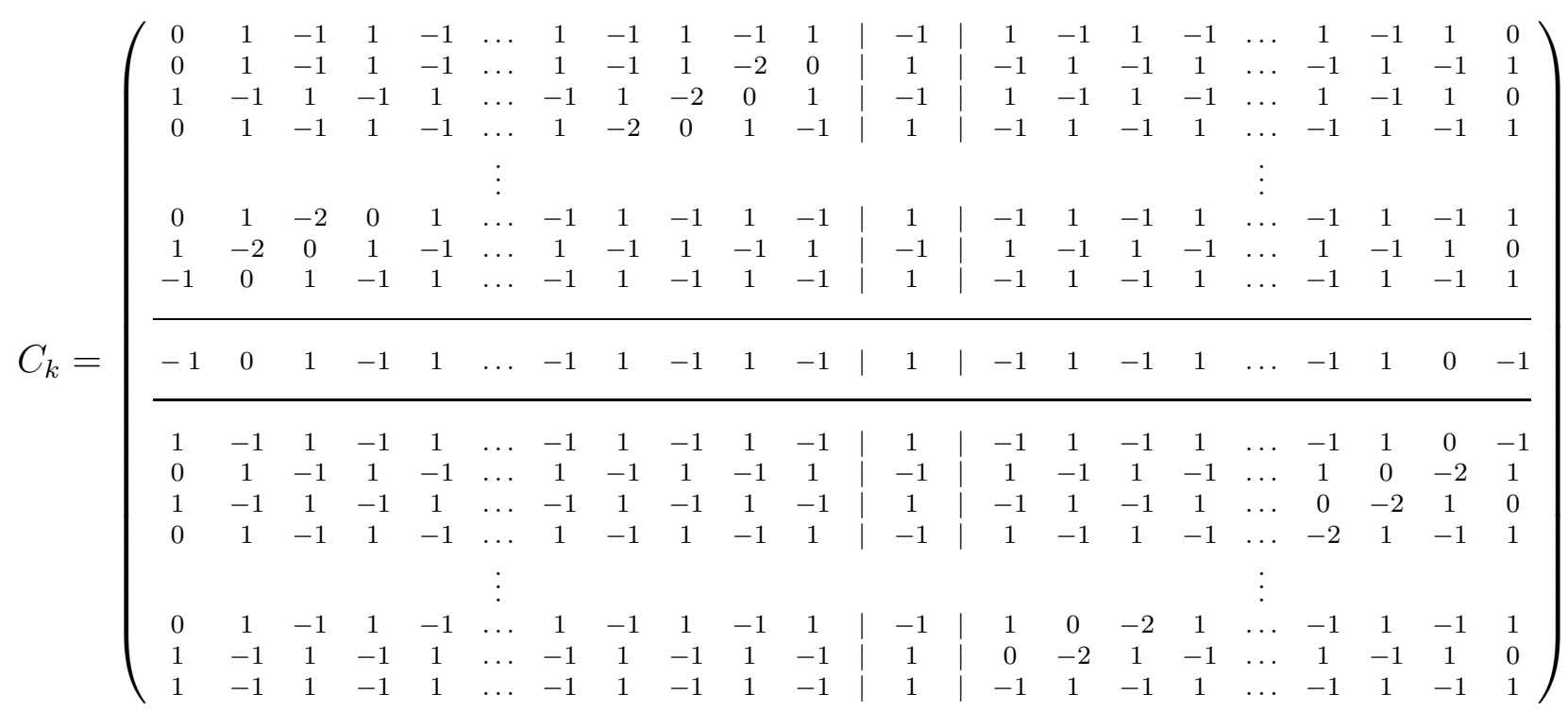

when $k \equiv 0 \bmod 4$. In each matrix the central row and column are flanked by four $k / 2 \times k / 2$ submatrices.

The next step is definition of the vector $\bar{\lambda}$. For this we will use the sequence of integers $\left(y_{k}\right)$ defined in Theorem 1, and also the well-known Pell numbers $\left(u_{k}\right)$, defined by: $u_{1}=1$, $u_{2}=2$, and $u_{i}=2 u_{i-1}+u_{i-2}$ for $i \geq 3$, so that $\left(u_{k}\right)=(1,2,5,12,29,70, \ldots)$. We will also need the initial value $u_{0}=0$ in some circumstances. In case that $k$ is even, we require yet another sequence of integers, closely related to the Pell numbers. Define $\left(v_{k}\right)$ by: $v_{i}=u_{i}+u_{i-1}$, so that

$$
\left(v_{1}, v_{2}, \ldots\right)=(1,3,7,17,41,99, \ldots) .
$$

See [5], for example, for information on these sequences.

The following results are routine, and their proofs are left to the reader.

Lemma 2 (a) $u_{2 n}=2 \sum_{i=1}^{n} u_{2 i-1}, \quad u_{2 n+1}=2 \sum_{i=1}^{n} u_{2 i}+1$.

(b) $u_{n+1}=\sum_{i=1}^{n} v_{i}+1$.

(c) $v_{n}=2 v_{n-1}+v_{n-2}$.

(d) $y_{n}= \begin{cases}2 \sum_{\substack{i=1 \\ n / 2}}^{n+1) / 2} u_{i} & \text { for } n \text { odd } \\ 2 \sum_{i=1}^{(n+1} u_{i}+u_{(n / 2)-1}+u_{n / 2} & \text { for } n \text { even } .\end{cases}$

(e) $y_{2 n}=2 \sum_{i=1}^{n} v_{i}+1$. 
Now define the $(k+1)$-vector

$$
\bar{\lambda}=\left\{\begin{array}{cc}
\left(u_{1}+\frac{k+1}{2}, u_{2}, u_{3}, \ldots, u_{(k+1) / 2}, u_{(k+1) / 2}, u_{(k-1) / 2}, \ldots, u_{2}, u_{1}+\frac{k+1}{2}\right)-\overline{1} & \text { for } k \text { odd }, \\
\left(u_{k / 2}+\frac{k}{2}+1, v_{2}, v_{3}, \ldots, v_{(k / 2)-1}, u_{(k / 2)-1}+1, u_{k / 2},\right. & \\
\left.u_{(k / 2)-1}+1, v_{(k / 2)-1}, v_{(k / 2)-2}, \ldots, v_{2}, \frac{k}{2}+1\right)-\overline{1} & \text { for } k \text { even. }
\end{array}\right.
$$

Note that, for $k$ odd,

$$
\sum \bar{\lambda}=2 \sum_{i=1}^{(k+1) / 2} u_{i}+(k+1)-(k+1)=2 \sum_{i=1}^{(k+1) / 2} u_{i}=y_{k}
$$

by Lemma 2(d), and for $k$ even,

$$
\begin{aligned}
\sum \bar{\lambda} & =\left(2 u_{k / 2}+k+4+2 \sum_{i=2}^{(k / 2)-1} v_{i}+2 u_{(k / 2)-1}\right)-(k+1) \\
& =2\left(u_{k / 2}+u_{(k / 2)-1}\right)+2 \sum_{i=2}^{(k / 2)-1} v_{i}+3 \\
& =2 \sum_{i=1}^{k / 2} v_{i}+1=y_{k},
\end{aligned}
$$

the last equality following from Lemma $2(\mathrm{e})$. So $\sum \bar{\lambda}=y_{k}$ for all $k$, and (2) becomes

$$
\begin{aligned}
\overline{0} & =\left(M_{k}-k s_{k} J_{k+1}\right)^{t} \bar{\lambda}=\left(M_{k}-\left[(k-1)+\frac{1}{y_{k}}\right] J_{k+1}\right)^{t} \bar{\lambda} \\
& =\left(C_{k}-\frac{1}{y_{k}} J_{k+1}\right)^{t} \bar{\lambda}=C_{k}^{t} \bar{\lambda}-\left(\frac{1}{y_{k}} \sum \bar{\lambda}\right) \overline{1}=C_{k}^{t} \bar{\lambda}-\overline{1}
\end{aligned}
$$

Thus we want to prove $C_{k}^{t} \bar{\lambda}=\overline{1}$.

We will do this by showing that the dot product of each column of $C_{k}$ with $\bar{\lambda}$ equals 1. Let $\operatorname{col}_{i}\left(C_{k}\right)$ denote the $i$ th column of $C_{k}$. Our proof treats the four congruence classes of $k$ modulo 4 separately.

Case (i): $k \equiv 1 \bmod 4$.

Note that, for each $i$, the $i$ th row of $C_{k}$ is the reverse of the $(k+2-i)$ th row by Lemma 1 , since the corresponding orientations are reverses of each other. Thus it is also true that $\operatorname{col}_{i}\left(C_{k}\right)$ is the reverse of $\operatorname{col}_{k+2-i}\left(C_{k}\right)$ for each $i$. Since $\bar{\lambda}$ is symmetric, $\operatorname{col}_{i}\left(C_{k}\right) \cdot \bar{\lambda}=$ 
$\operatorname{col}_{k+2-i}\left(C_{k}\right) \cdot \bar{\lambda}$, so we need only show that $\operatorname{col}_{i}\left(C_{k}\right) \cdot \bar{\lambda}=1$ for $1 \leq i \leq(k+1) / 2$. We first consider column 1 :

$$
\begin{aligned}
\operatorname{col}_{1}\left(C_{k}\right) \cdot \bar{\lambda}= & \left(u_{2}-1\right)+\left(u_{4}-1\right)+\cdots+\left(u_{(k-1) / 2}-1\right)-\left(u_{(k+1) / 2}-1\right) \\
& \quad+\left(u_{(k-1) / 2}-1\right)+\left(u_{(k-5) / 2}-1\right)+\cdots+\left(u_{2}-1\right)+\left(u_{1}+\frac{k-1}{2}\right) \\
= & 2 \sum_{i=1}^{(k-1) / 4}\left(u_{2 i}-1\right)-u_{(k+1) / 2}+1+u_{1}+\frac{k-1}{2} \\
= & 2 \sum_{i=1}^{(k-1) / 4} u_{2 i}-u_{(k+1) / 2}+2=1
\end{aligned}
$$

by Lemma 2(a). To handle the other columns, it seems easiest to show that $\left[\operatorname{col}_{i}\left(C_{k}\right)+\right.$ $\left.\operatorname{col}_{i+1}\left(C_{k}\right)\right] \cdot \bar{\lambda}=2$ for each $i \in\{1,2, \ldots,(k-1) / 2\}$. Since $\operatorname{col}_{1}\left(C_{k}\right) \cdot \bar{\lambda}=1$, it then follows that $\operatorname{col}_{i}\left(C_{k}\right) \cdot \bar{\lambda}=1$ for all $i \leq(k+1) / 2$ and thus for all $i$. A similar strategy will be adopted for the other three cases.

Thus we first note that

$$
\operatorname{col}_{1}\left(C_{k}\right)+\operatorname{col}_{2}\left(C_{k}\right)=(1,0,1,0, \ldots, 1,0,1,-1,-1 \mid 1,0,1,0, \ldots, 1,0,0)
$$

(where the midpoint of the $(k+1)$-vector is indicated by a vertical line), so

$$
\begin{aligned}
{\left[\operatorname{col}_{1}\left(C_{k}\right)+\operatorname{col}_{2}\left(C_{k}\right)\right] \cdot \bar{\lambda} } & =\left(u_{1}+\frac{k-1}{2}\right)+\left(u_{3}-1\right)+\left(u_{5}-1\right)+\cdots+\left(u_{(k-3) / 2}-1\right) \\
& -\left(u_{(k-1) / 2}-1\right)-\left(u_{(k+1) / 2}-1\right) \\
+\left(u_{(k+1) / 2}-1\right)+\left(u_{(k-3) / 2}-1\right)+\cdots+\left(u_{3}-1\right) & \\
= & 2 \sum_{i=2}^{(k-1) / 4}\left(u_{2 i-1}-1\right)-u_{(k-1) / 2}+1+u_{1}+\frac{k-1}{2} \\
= & 2 \sum_{i=1}^{(k-1) / 4} u_{2 i-1}-u_{(k-1) / 2}+2=2
\end{aligned}
$$

by Lemma $2(\mathrm{a})$. For any $i \in\{2,3, \ldots,(k-3) / 2\}$,

$$
\operatorname{col}_{i}\left(C_{k}\right)+\operatorname{col}_{i+1}\left(C_{k}\right)=(0,0, \ldots, 0,-1,-2, \stackrel{j}{1}, 0,0, \ldots, 0)
$$

where

$$
j=\frac{k+5}{2}-i
$$

Thus

$$
\begin{aligned}
{\left[\operatorname{col}_{i}\left(C_{k}\right)+\operatorname{col}_{i+1}\left(C_{k}\right)\right] \cdot \bar{\lambda} } & =-\left(u_{j-2}-1\right)-2\left(u_{j-1}-1\right)+\left(u_{j}-1\right) \\
& =u_{j}-2 u_{j-1}-u_{j-2}+2=2
\end{aligned}
$$


Finally,

$$
\operatorname{col}_{(k-1) / 2}\left(C_{k}\right)+\operatorname{col}_{(k+1) / 2}\left(C_{k}\right)=(0,-2,1,0,0, \ldots, 0),
$$

So

$$
\begin{aligned}
{\left[\operatorname{col}_{(k-1) / 2}\left(C_{k}\right)+\operatorname{col}_{(k+1) / 2}\left(C_{k}\right)\right] \cdot \bar{\lambda} } & =-2\left(u_{2}-1\right)+\left(u_{3}-1\right) \\
& =-2+4=2 .
\end{aligned}
$$

Case (ii): $k \equiv 3 \bmod 4$.

Once again we need only show that $\operatorname{col}_{i}\left(C_{k}\right) \cdot \bar{\lambda}=1$ for $1 \leq i \leq(k+1) / 2$. Proceeding as in Case (i), we first get

$$
\begin{aligned}
\operatorname{col}_{1}\left(C_{k}\right) \cdot \bar{\lambda}= & \left(u_{3}-1\right)+\left(u_{5}-1\right)+\cdots+\left(u_{(k-1) / 2}-1\right)-\left(u_{(k+1) / 2}-1\right) \\
& \quad+\left(u_{(k-1) / 2}-1\right)+\left(u_{(k-5) / 2}-1\right)+\cdots+\left(u_{3}-1\right)+\left(u_{1}+\frac{k-1}{2}\right) \\
= & 2 \sum_{i=2}^{(k+1) / 4}\left(u_{2 i-1}-1\right)-u_{(k+1) / 2}+1+u_{1}+\frac{k-1}{2} \\
= & 2 \sum_{i=1}^{(k+1) / 4} u_{2 i-1}-u_{(k+1) / 2}+1=1
\end{aligned}
$$

by Lemma 2(a). Then

$$
\operatorname{col}_{1}\left(C_{k}\right)+\operatorname{col}_{2}\left(C_{k}\right)=(1,1,0,1,0, \ldots, 1,0,1,-1,-1 \mid 1,0,1,0, \ldots, 0,1,0),
$$

so

$$
\begin{aligned}
{\left[\operatorname{col}_{1}\left(C_{k}\right)+\operatorname{col}_{2}\left(C_{k}\right)\right] \cdot \bar{\lambda}=} & \left(u_{1}+\frac{k-1}{2}\right)+\left(u_{2}-1\right)+\left(u_{4}-1\right)+\cdots+\left(u_{(k-3) / 2}-1\right) \\
-\left(u_{(k-1) / 2}-1\right)-\left(u_{(k+1) / 2}-1\right) & +\left(u_{(k+1) / 2}-1\right)+\left(u_{(k-3) / 2}-1\right)+\cdots+\left(u_{2}-1\right) \\
& =2 \sum_{i=1}^{(k-3) / 4}\left(u_{2 i}-1\right)-u_{(k-1) / 2}+1+u_{1}+\frac{k-1}{2} \\
= & 2 \sum_{i=1}^{(k-3) / 4} u_{2 i}-u_{(k-1) / 2}+3=2
\end{aligned}
$$

by Lemma 2(a). Now note that for any $i \in\{2,3, \ldots,(k-1) / 2\}, \operatorname{col}_{i}\left(C_{k}\right)+\operatorname{col}_{i+1}\left(C_{k}\right)$ in Case (ii) equals the sum of the same two columns in Case (i), so we must have

$$
\left[\operatorname{col}_{i}\left(C_{k}\right)+\operatorname{col}_{i+1}\left(C_{k}\right)\right] \cdot \bar{\lambda}=2
$$

for $2 \leq i \leq(k-1) / 2$ here as well, which completes the proof for this case. 
Case (iii): $k \equiv 2 \bmod 4$.

For this and the final case we must work a little harder, because we do not have quite the same symmetry in $C_{k}$ and $\bar{\lambda}$ when $k$ is even as we had when $k$ is odd.

First,

$$
\begin{aligned}
\operatorname{col}_{1}\left(C_{k}\right) \cdot \bar{\lambda}= & \left(v_{2}-1\right)+\left(v_{4}-1\right)+\cdots+\left(v_{(k / 2)-1}-1\right)-u_{(k / 2)-1}-\left(u_{k / 2}-1\right) \\
& +u_{(k / 2)-1}+\left(v_{(k / 2)-2}-1\right)+\left(v_{(k / 2)-4}-1\right)+\cdots+\left(v_{3}-1\right)+\frac{k}{2} \\
= & \sum_{i=2}^{(k / 2)-1}\left(v_{i}-1\right)-u_{k / 2}+1+\frac{k}{2} \\
= & \sum_{i=1}^{(k / 2)-1} v_{i}-u_{k / 2}+2=1
\end{aligned}
$$

by Lemma 2(b). Then

$$
\operatorname{col}_{1}\left(C_{k}\right)+\operatorname{col}_{2}\left(C_{k}\right)=(1,0,1,0, \ldots, 1,0,1,-1,-1|-1| 0,1,0,1, \ldots, 0,1,0),
$$

where the central $((k / 2)+1)$ st element has been sandwiched by vertical lines. So

$$
\begin{aligned}
{\left[\operatorname{col}_{1}\left(C_{k}\right)+\operatorname{col}_{2}\left(C_{k}\right)\right] \cdot \bar{\lambda}=} & \left(u_{k / 2}+\frac{k}{2}\right)+\left(v_{3}-1\right)+\left(v_{5}-1\right)+\cdots+\left(v_{(k / 2)-2}-1\right) \\
& -\left(v_{(k / 2)-1}-1\right)-u_{(k / 2)-1}-\left(u_{k / 2}-1\right) \\
& +\left(v_{(k / 2)-1}-1\right)+\left(v_{(k / 2)-3}-1\right)+\cdots+\left(v_{2}-1\right) \\
& =\sum_{i=2}^{(k / 2)-2}\left(v_{i}-1\right)-u_{(k / 2)-1}+\frac{k}{2}+1 \\
& =\sum_{i=1}^{(k / 2)-2} v_{i}-u_{(k / 2)-1}+3=2
\end{aligned}
$$

by Lemma 2(b). Also

$$
\operatorname{col}_{2}\left(C_{k}\right)+\operatorname{col}_{3}\left(C_{k}\right)=(0,0, \ldots, 0,-1,-2,1|1| 0,0, \ldots, 0),
$$

So

$$
\begin{aligned}
{\left[\operatorname{col}_{2}\left(C_{k}\right)+\operatorname{col}_{3}\left(C_{k}\right)\right] \cdot \bar{\lambda} } & =-\left(v_{(k / 2)-2}-1\right)-2\left(v_{(k / 2)-1}-1\right)+u_{(k / 2)-1}+\left(u_{k / 2}-1\right) \\
& =-v_{(k / 2)-2}-2 v_{(k / 2)-1}+\left(u_{(k / 2)-1}+u_{k / 2}\right)+2 \\
& =v_{k / 2}-2 v_{(k / 2)-1}-v_{(k / 2)-2}+2=2
\end{aligned}
$$

by Lemma 2(c). For any $i \in\{3,4, \ldots,(k / 2)-2\}$,

$$
\operatorname{col}_{i}\left(C_{k}\right)+\operatorname{col}_{i+1}\left(C_{k}\right)=\left(0,0, \ldots, 0,-1,-2,{ }_{1}^{j}, 0,0, \ldots, 0\right)
$$


where

$$
j=\frac{k}{2}+2-i
$$

and we get

$$
\begin{aligned}
{\left[\operatorname{col}_{i}\left(C_{k}\right)+\operatorname{col}_{i+1}\left(C_{k}\right)\right] \cdot \bar{\lambda} } & =-\left(v_{j-2}-1\right)-2\left(v_{j-1}-1\right)+\left(v_{j}-1\right) \\
& =v_{j}-2 v_{j-1}-v_{j-2}+2=2
\end{aligned}
$$

by Lemma 2(c). Next

$$
\operatorname{col}_{(k / 2)-1}\left(C_{k}\right)+\operatorname{col}_{k / 2}\left(C_{k}\right)=(0,-2,1,0,0, \ldots, 0),
$$

so

$$
\begin{aligned}
{\left[\operatorname{col}_{(k / 2)-1}\left(C_{k}\right)+\operatorname{col}_{k / 2}\left(C_{k}\right)\right] \cdot \bar{\lambda} } & =-2\left(v_{2}-1\right)+\left(v_{3}-1\right) \\
& =-4+6=2 .
\end{aligned}
$$

And one more:

$$
\operatorname{col}_{k / 2}\left(C_{k}\right)+\operatorname{col}_{(k / 2)+1}\left(C_{k}\right)=(0,1,0,0, \ldots, 0)
$$

So

$$
\left[\operatorname{col}_{k / 2}\left(C_{k}\right)+\operatorname{col}_{(k / 2)+1}\left(C_{k}\right)\right] \cdot \bar{\lambda}=v_{2}-1=2 .
$$

Now notice that for $i \in\{2,3, \ldots, k / 2\}$,

$$
\operatorname{col}_{k+2-i}\left(C_{k}\right)+\operatorname{col}_{k+1-i}\left(C_{k}\right) \text { is the reverse of } \operatorname{col}_{i}\left(C_{k}\right)+\operatorname{col}_{i+1}\left(C_{k}\right) \text {. }
$$

Since the first and last entries of $\operatorname{col}_{i}\left(C_{k}\right)+\operatorname{col}_{i+1}\left(C_{k}\right)$ equal zero for all $2 \leq i \leq k / 2$, and since $\bar{\lambda}$ is symmetric except for its first and last entries, it follows that

$$
\left[\operatorname{col}_{k+2-i}\left(C_{k}\right)+\operatorname{col}_{k+1-i}\left(C_{k}\right)\right] \cdot \bar{\lambda}=\left[\operatorname{col}_{i}\left(C_{k}\right)+\operatorname{col}_{i+1}\left(C_{k}\right)\right] \cdot \bar{\lambda}=2
$$

for $2 \leq i \leq k / 2$. This means that we will be done with this case once we verify that

$$
\left[\operatorname{col}_{k}\left(C_{k}\right)+\operatorname{col}_{k+1}\left(C_{k}\right)\right] \cdot \bar{\lambda}=2 \quad \text { and } \quad \operatorname{col}_{k+1}\left(C_{k}\right) \cdot \bar{\lambda}=1 .
$$

So what are we waiting for?

$$
\operatorname{col}_{k}\left(C_{k}\right)+\operatorname{col}_{k+1}\left(C_{k}\right)=(1,1,0,1,0, \ldots, 1,0|-1|-1,-1,1,0,1,0, \ldots, 1,0,0),
$$

SO

$$
\begin{aligned}
{\left[\operatorname{col}_{k}\left(C_{k}\right)+\operatorname{col}_{k+1}\left(C_{k}\right)\right] \cdot \bar{\lambda}=} & \left(u_{k / 2}+\frac{k}{2}\right)+\left(v_{2}-1\right)+\left(v_{4}-1\right)+\cdots+\left(v_{(k / 2)-1}-1\right) \\
& -\left(u_{k / 2}-1\right)-u_{(k / 2)-1}-\left(v_{(k / 2)-1}-1\right) \\
& +\left(v_{(k / 2)-2}-1\right)+\left(v_{(k / 2)-4}-1\right)+\cdots+\left(v_{3}-1\right) \\
= & \sum_{i=2}^{(k / 2)-2}\left(v_{i}-1\right)-u_{(k / 2)-1}+\frac{k}{2}+1 \\
= & \sum_{i=1}^{(k / 2)-2} v_{i}-u_{(k / 2)-1}+3=2
\end{aligned}
$$


by Lemma 2(b). Finally,

$$
\begin{aligned}
\operatorname{col}_{k+1}\left(C_{k}\right) \cdot \bar{\lambda}= & \left(v_{3}-1\right)+\left(v_{5}-1\right)+\cdots+\left(v_{(k / 2)-2}-1\right)+u_{(k / 2)-1}-\left(u_{k / 2}-1\right) \\
& -u_{(k / 2)-1}+\left(v_{(k / 2)-1}-1\right)+\left(v_{(k / 2)-3}-1\right)+\cdots+\left(v_{2}-1\right)+\frac{k}{2} \\
= & \sum_{i=2}^{(k / 2)-1}\left(v_{i}-1\right)-u_{k / 2}+1+\frac{k}{2} \\
= & \sum_{i=1}^{(k / 2)-1} v_{i}-u_{k / 2}+2=1
\end{aligned}
$$

by Lemma 2(b).

Case (iv): $k \equiv 0 \bmod 4$.

This case works almost the same way as Case (iii). For column 1,

$$
\begin{aligned}
\operatorname{col}_{1}\left(C_{k}\right) \cdot \bar{\lambda}= & \left(v_{3}-1\right)+\left(v_{5}-1\right)+\cdots+\left(v_{(k / 2)-1}-1\right)-u_{(k / 2)-1}-\left(u_{k / 2}-1\right) \\
& +u_{(k / 2)-1}+\left(v_{(k / 2)-2}-1\right)+\left(v_{(k / 2)-4}-1\right)+\cdots+\left(v_{2}-1\right)+\frac{k}{2} \\
= & \sum_{i=2}^{(k / 2)-1}\left(v_{i}-1\right)-u_{k / 2}+1+\frac{k}{2} \\
= & \sum_{i=1}^{(k / 2)-1} v_{i}-u_{k / 2}+2=1
\end{aligned}
$$

by Lemma 2(b). Then

$$
\operatorname{col}_{1}\left(C_{k}\right)+\operatorname{col}_{2}\left(C_{k}\right)=(1,1,0,1,0, \ldots, 1,0,1,-1,-1|-1| 0,1,0,1, \ldots, 0,1,0,0),
$$

so

$$
\begin{aligned}
{\left[\operatorname{col}_{1}\left(C_{k}\right)+\operatorname{col}_{2}\left(C_{k}\right)\right] \cdot \bar{\lambda}=} & \left(u_{k / 2}+\frac{k}{2}\right)+\left(v_{2}-1\right)+\left(v_{4}-1\right)+\cdots+\left(v_{(k / 2)-2}-1\right) \\
& -\left(v_{(k / 2)-1}-1\right)-u_{(k / 2)-1}-\left(u_{k / 2}-1\right) \\
& +\left(v_{(k / 2)-1}-1\right)+\left(v_{(k / 2)-3}-1\right)+\cdots+\left(v_{3}-1\right) \\
= & \sum_{i=2}^{(k / 2)-2}\left(v_{i}-1\right)-u_{(k / 2)-1}+\frac{k}{2}+1 \\
= & \sum_{i=1}^{(k / 2)-2} v_{i}-u_{(k / 2)-1}+3=2
\end{aligned}
$$

by Lemma 2(b). But now we have that for any $i \in\{2,3, \ldots, k-1\}, \operatorname{col}_{i}\left(C_{k}\right)+\operatorname{col}_{i+1}\left(C_{k}\right)$ in Case (iv) equals the sum of the same two columns in Case (iii), so

$$
\left[\operatorname{col}_{i}\left(C_{k}\right)+\operatorname{col}_{i+1}\left(C_{k}\right)\right] \cdot \bar{\lambda}=2
$$


for $2 \leq i \leq k-1$ in this case too. Thus we once again need only show

$$
\left[\operatorname{col}_{k}\left(C_{k}\right)+\operatorname{col}_{k+1}\left(C_{k}\right)\right] \cdot \bar{\lambda}=2 \quad \text { and } \quad \operatorname{col}_{k+1}\left(C_{k}\right) \cdot \bar{\lambda}=1 .
$$

This time,

$$
\operatorname{col}_{k}\left(C_{k}\right)+\operatorname{col}_{k+1}\left(C_{k}\right)=(1,0,1,0, \ldots, 1,0|-1|-1,-1,1,0,1,0, \ldots, 1,0)
$$

results in

$$
\begin{aligned}
{\left[\operatorname{col}_{k}\left(C_{k}\right)+\operatorname{col}_{k+1}\left(C_{k}\right)\right] \cdot \bar{\lambda}=} & \left(u_{k / 2}+\frac{k}{2}\right)+\left(v_{3}-1\right)+\left(v_{5}-1\right)+\cdots+\left(v_{(k / 2)-1}-1\right) \\
& -\left(u_{k / 2}-1\right)-u_{(k / 2)-1}-\left(v_{(k / 2)-1}-1\right) \\
& +\left(v_{(k / 2)-2}-1\right)+\left(v_{(k / 2)-4}-1\right)+\cdots+\left(v_{2}-1\right) \\
= & \sum_{i=2}^{(k / 2)-2}\left(v_{i}-1\right)-u_{(k / 2)-1}+\frac{k}{2}+1 \\
= & \sum_{i=1}^{(k / 2)-2} v_{i}-u_{(k / 2)-1}+3=2
\end{aligned}
$$

by Lemma $2(\mathrm{~b})$, and

$$
\begin{aligned}
\operatorname{col}_{k+1}\left(C_{k}\right) \cdot \bar{\lambda}= & \left(v_{2}-1\right)+\left(v_{4}-1\right)+\cdots+\left(v_{(k / 2)-2}-1\right)+u_{(k / 2)-1}-\left(u_{k / 2}-1\right) \\
& -u_{(k / 2)-1}+\left(v_{(k / 2)-1}-1\right)+\left(v_{(k / 2)-3}-1\right)+\cdots+\left(v_{3}-1\right)+\frac{k}{2} \\
= & \sum_{i=2}^{(k / 2)-1}\left(v_{i}-1\right)-u_{k / 2}+1+\frac{k}{2} \\
= & \sum_{i=1}^{(k / 2)-1} v_{i}-u_{k / 2}+2=1
\end{aligned}
$$

by Lemma 2(b). This finishes the last case, and the verification of the lower bound in Theorem 1.

\section{The upper bound}

Suppose that a $k$-antichain $A$ in $L=\mathbf{k} \times \mathbf{n}$ is defined by the $(k+1)$-vector $\bar{n}$ (so $\sum \bar{n}=n$ ). We noted in Section 2 that the number of elements of $L$ captured by an orientation $o$ of $A$ is $\bar{v}_{o} \cdot \bar{n}+r_{0}$, where $r_{0} \leq k$. Thus to prove $s_{k}\left(\mathcal{G}_{k}\right) \leq s_{k}$ for any fixed $k$, where $s_{k}$ is given at the beginning of $\S 3$, it is enough to verify that, for each $\epsilon>0$, there is some $\bar{n}$ depending on $\epsilon$ so that

$$
\bar{v}_{o} \cdot \bar{n}+r_{0} \leq\left(s_{k}+\epsilon\right)(k n)
$$

for all $2^{k}$ orientations $o$ of the $k$-antichain $A$ associated with $\bar{n}$. 
For this we use a particular symmetric $(k+1)$-vector $\bar{n}_{k}(k \geq 1)$, namely

$$
\bar{n}_{k}=\left\{\begin{array}{ll}
\left(u_{1}, u_{2}, \ldots, u_{(k+1) / 2}, u_{(k+1) / 2}, u_{(k-1) / 2}, \ldots, u_{2}, u_{1}\right) & \text { for } k \text { odd, } \\
\left(u_{1}, u_{2}, \ldots, u_{k / 2}, u_{k / 2}+u_{(k / 2)-1}, u_{k / 2}, u_{(k / 2)-1}, \ldots, u_{2}, u_{1}\right) & \text { for } k \text { even. }
\end{array}\right\}
$$

Note the remarkable similarity between $\bar{n}_{k}$ and $\bar{\lambda}(\S 3)$ in the case that $k$ is odd. Note also that, for all $k$, the sum of the entries of $\bar{n}_{k}$ equals precisely $y_{k}$, by Lemma $2(\mathrm{~d})$.

In fact, we prove that for all $k$, the vector $\bar{n}_{k}$ satisfies

$$
\bar{v}_{o} \cdot \bar{n}_{k} \leq s_{k}\left(k y_{k}\right)
$$

for all $2^{k}$ orientations $o$ of $A$. Then for any positive integer $t$, the vector $\bar{n}=t \bar{n}_{k}$, corresponding to a maximal $k$-antichain $A$ in $\mathbf{k} \times \mathbf{n}$ (where $n=\sum \bar{n}=t y_{k}$ ), satisfies

$$
\bar{v}_{o} \cdot \bar{n} \leq s_{k}\left(k t y_{k}\right)=s_{k}(k n)
$$

and thus

$$
\bar{v}_{o} \cdot \bar{n}+r_{0} \leq s_{k}(k n)+k=\left(s_{k}+\frac{1}{t y_{k}}\right)(k n)
$$

for all $2^{k}$ orientations $o$ of $A$. Letting $t \rightarrow \infty$, we are done.

We prove (5) by showing that

- for each $o$, there is an alternating orientation $a$ [one of the four orientations defined in (3)] such that

$$
\bar{v}_{o} \cdot \bar{n}_{k} \leq \bar{v}_{a} \cdot \bar{n}_{k}
$$

and

- for every alternating orientation $a$,

$$
\bar{v}_{a} \cdot \bar{n}_{k}=s_{k}\left(k y_{k}\right) .
$$

Our proof of (6) requires that we alter orientations in a stepwise fashion. Suppose $o$ is not an alternating orientation. So, upon identifying $o$ with a $k$-sequence of $\uparrow$ 's and $\downarrow$ 's, we see that $o$ has two consecutive $\uparrow$ 's or two consecutive $\downarrow$ 's. We know that $\bar{n}_{k}$ is symmetric, and we have that $\bar{v}_{o} \cdot \bar{n}_{k}=\bar{v}_{o^{r}} \cdot \bar{n}_{k}$, by Lemma 1 . Therefore we may assume that $o$ has two $\uparrow$ 's in positions $r-1$ and $r$, where

Case (i): $r \leq(k+1) / 2$ and the first $r-1$ entries of $o$ are alternating, or

Case (ii): $r-1 \geq(k+1) / 2$ and the last $k-(r-1)$ entries of $o$ are alternating, or

Case (iii): $k$ is even, $r=k / 2+1$, and $o$ is alternating except for consecutive $\uparrow$ 's in positions $r-1$ and $r$. 
In each of (i) - (iii), and depending upon the parity of $k$ and $r$, we shall define a new orientation $\hat{o}$ by reversing all the arrows in the first $r-1$ positions [in Cases (i) and (iii)] or all arrows after and including the $r^{\text {th }}$ position [in Case (ii)]. This is made precise in each case following. Set $\bar{v}_{o}=\left(a_{1}, \ldots, a_{k+1}\right)$ and $\bar{v}_{\hat{o}}=\left(b_{1}, \ldots, b_{k+1}\right)$.

Assume that Case (i) holds and $r$ is odd. Then $o$ and $\hat{o}$ are of this form:
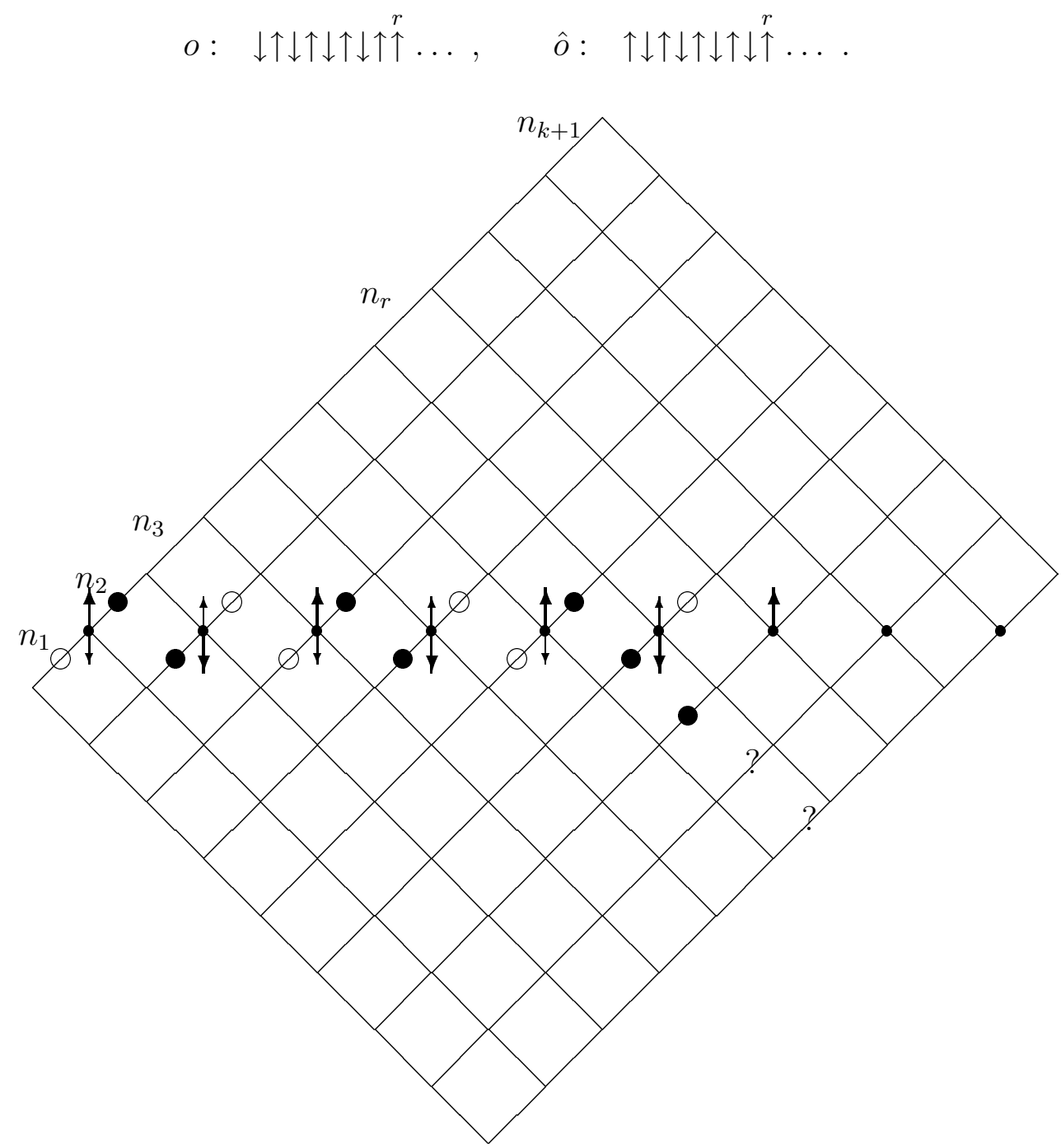

Figure 4: intervals lost and gained in switching from $o$ to $\hat{o}$

See Figure 4, where $o$ is indicated with shorter arrows and $\hat{o}$ with longer arrows (where they differ); hollow circles indicate intervals captured by o but not $\hat{o}$; solid circles indicate intervals captured by $\hat{o}$ but not $o$; and question marks are intervals that are captured by $\hat{o}$ and may or may not have been captured by $o$. It is easy to see that $b_{t}=a_{t}$ for all $t \geq r+1$, and

$$
b_{r}=a_{r}-1, \quad b_{r-1} \geq a_{r-1}+3, \quad b_{r-2}=a_{r-2}-2, \quad b_{r-3}=a_{r-3}+2, \quad \ldots,
$$




$$
b_{3}=a_{3}-2, \quad b_{2}=a_{2}+2, \quad b_{1}=a_{1}-1 .
$$

Therefore, by Lemma 2 (a) and the Pell recurrence $u_{i}=2 u_{i-1}+u_{i-2}$ (and since the components of $\bar{n}_{k}$ are all positive),

$$
\begin{aligned}
\bar{v}_{\hat{o}} \cdot \bar{n}_{k}-\bar{v}_{o} \cdot \bar{n}_{k} & =\left(\bar{v}_{\hat{o}}-\bar{v}_{o}\right) \cdot \bar{n}_{k} \\
& \geq(-1,2,-2,2, \ldots,-2,3,-1,0, \ldots, 0) \cdot \bar{n}_{k} \\
& =-u_{1}+2 u_{2}-2 u_{3}+2 u_{4}-\cdots+2 u_{r-3}-2 u_{r-2}+3 u_{r-1}-u_{r} \\
& =-2\left(u_{1}+u_{3}+\cdots+u_{r}\right)+u_{1}+u_{r}+2\left(u_{2}+u_{4}+\cdots+u_{r-1}\right)+u_{r-1} \\
& =-u_{r+1}+u_{1}+u_{r}+u_{r}-1+u_{r-1} \\
& =0 .
\end{aligned}
$$

On the other hand, if Case (i) holds and $r$ is even, then $o$ and $\hat{o}$ are of the form:

$$
o: \quad \uparrow \downarrow \uparrow \downarrow \uparrow \downarrow \uparrow \downarrow \uparrow \uparrow \ldots, \quad \hat{o}: \quad \downarrow \uparrow \uparrow \uparrow \downarrow \uparrow \downarrow \uparrow \downarrow \uparrow \ldots .
$$

Then $b_{t}=a_{t}$ for all $t \geq r+1$, and

$$
\begin{gathered}
b_{r}=a_{r}-1, \quad b_{r-1} \geq a_{r-1}+3, \quad b_{r-2}=a_{r-2}-2, \quad b_{r-3}=a_{r-3}+2, \quad \ldots, \\
b_{3}=a_{3}+2, \quad b_{2}=a_{2}-2, \quad b_{1}=a_{1}+1 .
\end{gathered}
$$

Therefore

$$
\begin{aligned}
\bar{v}_{\hat{o}} \cdot \bar{n}_{k}-\bar{v}_{o} \cdot \bar{n}_{k} & =\left(\bar{v}_{\hat{o}}-\bar{v}_{o}\right) \cdot \bar{n}_{k} \\
& \geq(1,-2,2,-2, \ldots,-2,3,-1,0, \ldots, 0) \cdot \bar{n}_{k} \\
& =u_{1}-2 u_{2}+2 u_{3}-\cdots+2 u_{r-3}-2 u_{r-2}+3 u_{r-1}-u_{r} \\
& =2\left(u_{1}+u_{3}+\cdots+u_{r-1}\right)-u_{1}+u_{r-1}-2\left(u_{2}+u_{4}+\cdots+u_{r}\right)+u_{r} \\
& =u_{r}-u_{1}+u_{r-1}-u_{r+1}+1+u_{r} \\
& =0 .
\end{aligned}
$$

Next, assume that Case (ii) holds. If $k-r$ is even, then $o$ and $\hat{o}$ are of the form:

$$
o: \quad \ldots \uparrow \uparrow \uparrow \uparrow \uparrow \uparrow \uparrow \uparrow, \quad \hat{o}: \quad \ldots \uparrow \uparrow^{r} \uparrow \downarrow \uparrow \uparrow \downarrow \uparrow \downarrow .
$$

This time we have $b_{t} \geq a_{t}$ for all $t \leq r-2$, and

$$
\begin{gathered}
b_{r-1}=a_{r-1}+1, \quad b_{r}=a_{r}+1, \quad b_{r+1}=a_{r+1}-2, \quad b_{r+2}=a_{r+2}+2, \quad \ldots, \\
b_{k-1}=a_{k-1}-2, \quad b_{k}=a_{k}+2, \quad b_{k+1}=a_{k+1}-1 .
\end{gathered}
$$

Therefore, since for $(k+3) / 2 \leq t \leq k+1$ the $t^{\text {th }}$ component of $\bar{n}_{k}$ is $u_{k-t+2}$, we get

$$
\begin{aligned}
\bar{v}_{\hat{o}} \cdot \bar{n}_{k}-\bar{v}_{o} \cdot \bar{n}_{k} & =\left(\bar{v}_{\hat{o}}-\bar{v}_{o}\right) \cdot \bar{n}_{k} \\
& \geq(0,0, \ldots, 0,1, \stackrel{r}{1},-2,2, \ldots,-2,2,-1) \cdot \bar{n}_{k} \\
& =w+u_{k-r+2}-2 u_{k-r+1}+2 u_{k-r}-\cdots-2 u_{3}+2 u_{2}-u_{1}
\end{aligned}
$$


where

$$
w= \begin{cases}u_{k-r+3} & \text { if } r>(k+4) / 2 \\ u_{k / 2}+u_{(k / 2)-1} & \text { if } k \text { is even and } r=(k+4) / 2 \\ u_{(k+1) / 2} & \text { if } k \text { is odd and } r=(k+3) / 2\end{cases}
$$

Thus

$$
\begin{aligned}
\bar{v}_{\hat{o}} \cdot \bar{n}_{k} & -\bar{v}_{o} \cdot \bar{n}_{k} \\
& \geq-2\left(u_{1}+u_{3}+\cdots+u_{k-r+1}\right)+u_{1}+2\left(u_{2}+u_{4}+\cdots+u_{k-r+2}\right)-u_{k-r+2}+w \\
& =-u_{k-r+2}+u_{1}+u_{k-r+3}-1-u_{k-r+2}+w \\
& =u_{k-r+1}+w>0
\end{aligned}
$$

again applying the recurrence relation and Lemma 2(a).

If Case (ii) holds and $k-r$ is odd, then

$$
o: \quad \ldots \uparrow \uparrow \uparrow^{r} \downarrow \uparrow \downarrow \uparrow \downarrow, \quad \hat{o}: \quad \ldots \uparrow^{r} \downarrow \uparrow \downarrow \uparrow \downarrow \uparrow .
$$

We have $b_{t} \geq a_{t}$ for all $t \leq r-2$, and

$$
\begin{gathered}
b_{r-1}=a_{r-1}+1, \quad b_{r}=a_{r}+1, \quad b_{r+1}=a_{r+1}-2, \quad b_{r+2}=a_{r+2}+2, \quad \ldots, \\
b_{k-1}=a_{k-1}+2, \quad b_{k}=a_{k}-2, \quad b_{k+1}=a_{k+1}+1 .
\end{gathered}
$$

Therefore we get

$$
\begin{aligned}
\bar{v}_{\hat{o}} \cdot \bar{n}_{k}-\bar{v}_{o} \cdot \bar{n}_{k} & =\left(\bar{v}_{\hat{o}}-\bar{v}_{o}\right) \cdot \bar{n}_{k} \\
& \geq(0,0, \ldots, 0,1, \stackrel{r}{1},-2,2, \ldots, 2,-2,1) \cdot \bar{n}_{k} \\
& =w+u_{k-r+2}-2 u_{k-r+1}+2 u_{k-r}-\cdots+2 u_{3}-2 u_{2}+u_{1}
\end{aligned}
$$

with $w$ defined as above. Thus once again

$$
\begin{aligned}
\bar{v}_{\hat{o}} \cdot \bar{n}_{k} & -\bar{v}_{o} \cdot \bar{n}_{k} \\
& \geq 2\left(u_{1}+u_{3}+\cdots+u_{k-r+2}\right)-u_{1}-u_{k-r+2}-2\left(u_{2}+u_{4}+\cdots+u_{k-r+1}\right)+w \\
& =u_{k-r+3}-u_{1}-u_{k-r+2}-u_{k-r+2}+1+w \\
& =u_{k-r+1}+w>0 .
\end{aligned}
$$

Finally, assume that Case (iii) holds. In this case, we reverse the first $k / 2$ arrows in $o$ to obtain $\hat{o}$, so the latter is an alternating sequence. Then $b_{t}=a_{t}$ for all $t \geq k / 2+2$, and $b_{k / 2+1}=a_{k / 2+1}-1, \quad b_{k / 2}=a_{k / 2}+3, \quad b_{k / 2-1}=a_{k / 2-1}-2, \quad b_{k / 2-2}=a_{k / 2-2}+2, \quad \ldots$, ending with

$$
b_{3}=a_{3}+2, \quad b_{2}=a_{2}-2, \quad b_{1}=a_{1}+1 \quad(k / 2 \text { odd })
$$


or

$$
b_{3}=a_{3}-2, \quad b_{2}=a_{2}+2, \quad b_{1}=a_{1}-1 \quad(k / 2 \text { even }) .
$$

The case is finished with two more easy calculations: if $k / 2$ is odd, then

$$
\begin{aligned}
\bar{v}_{\hat{o}} \cdot \bar{n}_{k}-\bar{v}_{o} \cdot \bar{n}_{k} & =\left(\bar{v}_{\hat{o}}-\bar{v}_{o}\right) \cdot \bar{n}_{k} \\
& =(1,-2,2, \ldots,-2,3,-1,0, \ldots, 0) \cdot \bar{n}_{k} \\
& =u_{1}-2 u_{2}+2 u_{3}-\cdots-2 u_{(k / 2)-1}+3 u_{k / 2}-\left(u_{k / 2}+u_{(k / 2)-1}\right) \\
& =2\left(u_{1}+u_{3}+\cdots+u_{k / 2}\right)-u_{1}-2\left(u_{2}+u_{4}+\cdots+u_{(k / 2)-1}\right)-u_{(k / 2)-1} \\
& =u_{(k / 2)+1}-u_{1}-u_{k / 2}+1-u_{(k / 2)-1} \\
& =u_{k / 2}>0 ;
\end{aligned}
$$

and if $k / 2$ is even then

$$
\begin{aligned}
\bar{v}_{\hat{o}} \cdot \bar{n}_{k}-\bar{v}_{o} \cdot \bar{n}_{k} & =\left(\bar{v}_{\hat{o}}-\bar{v}_{o}\right) \cdot \bar{n}_{k} \\
& =(-1,2,-2, \ldots,-2,3,-1,0, \ldots, 0) \cdot \bar{n}_{k} \\
& =-u_{1}+2 u_{2}-2 u_{3}+\cdots-2 u_{(k / 2)-1}+3 u_{k / 2}-\left(u_{k / 2}+u_{(k / 2)-1}\right) \\
& =-2\left(u_{1}+u_{3}+\cdots+u_{(k / 2)-1}\right)+u_{1}+2\left(u_{2}+u_{4}+\cdots+u_{k / 2}\right)-u_{(k / 2)-1} \\
& =-u_{k / 2}+u_{1}+u_{(k / 2)+1}-1-u_{(k / 2)-1} \\
& =u_{k / 2}>0 .
\end{aligned}
$$

By successively applying Cases (i) - (iii), and appealing to symmetry to deal with consecutive $\downarrow$ 's, we have verified (6).

Note that, by the above proof, equality can only hold in (6) in Case (i), and then only for orientations $o$ such that $b_{r-1}=a_{r-1}+3$, where $a_{r-1}$ and $b_{r-1}$ are the $(r-1)$ th components of the capture vectors of $o$ and $\hat{o}$ respectively. The reader can check that this happens precisely if the two consecutive $\uparrow$ 's in $O$ are followed by a $\downarrow$.

Let's turn to the proof of (7), namely that $\bar{v}_{a} \cdot \bar{n}_{k}=s_{k}\left(k y_{k}\right)$ for all alternating orientations $a$. First notice that $s_{k}\left(k y_{k}\right)=(k-1) y_{k}+1$. The capture vectors of the alternating orientations are given in Section 3. For $k$ odd, one vector is the reverse of the other, so, since $\bar{n}_{k}$ is symmetric, we need only check one, say $a_{\uparrow}$ :

$$
\begin{aligned}
\bar{v}_{a_{\uparrow}} \cdot \bar{n}_{k} & =(\overline{k-1}+(0,1,-1,1,-1, \ldots,-1,1)) \cdot \bar{n}_{k} \\
& =(k-1) y_{k}+1,
\end{aligned}
$$

because $\sum \bar{n}_{k}=y_{k}$ and all terms of the second dot product cancel except the last term of $\bar{n}_{k}$.

For $k$ even, we first consider orientation $a=a_{\uparrow}$ and $k \equiv 0 \bmod 4$ :

$$
\begin{aligned}
\bar{v}_{a_{\uparrow}} \cdot \bar{n}_{k}= & (\overline{k-1}+(0,1,-1,1,-1, \ldots,-1,1,0)) \cdot \bar{n}_{k} \\
= & (k-1) y_{k}+2\left(u_{2}+u_{4}+\cdots+u_{k / 2}\right)-2\left(u_{1}+u_{3}+u_{5}+\cdots+u_{(k-2) / 2}\right) \\
& +2 u_{1}-\left(u_{(k-2) / 2}+u_{k / 2}\right) \\
& =(k-1) y_{k}+u_{(k+2) / 2}-1-u_{k / 2}+2-u_{(k-2) / 2}-u_{k / 2} \\
& =(k-1) y_{k}+1,
\end{aligned}
$$


using Lemma 2(a) and the recurrence defining $u_{k}$. The case with $k \equiv 2 \bmod 4$ is just about identical:

$$
\begin{aligned}
\bar{v}_{a_{\uparrow}} \cdot \bar{n}_{k} & =(\overline{k-1}+(0,1,-1,1,-1, \ldots,-1,1,0)) \cdot \bar{n}_{k} \\
& =(k-1) y_{k}+2\left(u_{2}+u_{4}+\cdots+u_{(k / 2)-1}\right)-2\left(u_{1}+u_{3}+u_{5}+\cdots+u_{k / 2}\right) \\
& +2 u_{1}+\left(u_{(k-2) / 2}+u_{k / 2}\right) \\
& =(k-1) y_{k}+u_{k / 2}-1-u_{(k / 2)+1}+2+u_{(k-2) / 2}+u_{k / 2} \\
& =(k-1) y_{k}+1 .
\end{aligned}
$$

Now consider the orientation $a=a_{\downarrow}$. If $k \equiv 0 \bmod 4$ :

$$
\begin{aligned}
\bar{v}_{a_{\downarrow}} \cdot \bar{n}_{k} & =(\overline{k-1}+(1,-1,1,-1, \ldots,-1,1)) \cdot \bar{n}_{k} \\
& =(k-1) y_{k}+2\left(u_{1}+u_{3}+\cdots+u_{(k-2) / 2}\right)-2\left(u_{2}+u_{4}+\cdots+u_{k / 2}\right) \\
& +\left(u_{(k-2) / 2}+u_{k / 2}\right) \\
& =(k-1) y_{k}+u_{k / 2}-u_{(k+2) / 2}+1+u_{(k-2) / 2}+u_{k / 2} \\
& =(k-1) y_{k}+1,
\end{aligned}
$$

again using Lemma $2(\mathrm{a})$ and the recurrence defining $u_{k}$. The case with $k \equiv 2 \bmod 4$ is the same:

$$
\begin{aligned}
\bar{v}_{a_{\downarrow}} \cdot \bar{n}_{k} & =(\overline{k-1}+(1,-1,1,-1, \ldots,-1,1)) \cdot \bar{n}_{k} \\
& =(k-1) y_{k}+2\left(u_{1}+u_{3}+\cdots+u_{k / 2}\right)-2\left(u_{2}+u_{4}+\cdots+u_{(k / 2)-1}\right) \\
& =(k-1) y_{k}+u_{(k / 2)+1}-u_{k / 2}+1-u_{(k / 2)-1}-u_{k / 2} \\
& =(k-1) y_{k}+1 .
\end{aligned}
$$

This completes the proof of (7) and verification of the upper bound. The proof of Theorem 1 is complete.

Note that, by (7) and the remark following the proof of (6), all the orientations $o$ in the families $\mathcal{O}$ defined in $\S 3$ satisfy $\bar{v}_{o} \cdot \bar{n}_{k}=s_{k}\left(k y_{k}\right)$ and so capture essentially the maximum number of elements of the lattice defined by the vector $\bar{n}_{k}$. This helps to explain how we chose these orientations. It has also helped to suggest an open problem (Problem 3) recorded in $\S 6$.

Also note that, as we observed in [3], the vectors $\bar{n}_{k}$ can be arranged in a Pascal-like triangle (defining $\bar{n}_{0}=(1)$ as the first row):

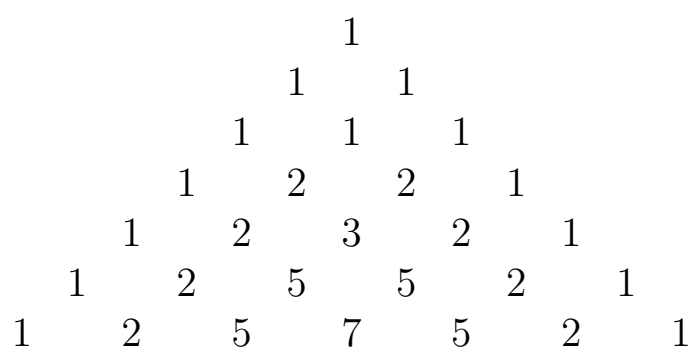




\section{$5 \quad$ Uniqueness of $\bar{n}_{k}$ for $k$ odd}

Here we establish that for odd $k$, the vector $\bar{n}_{k}$ defined in the previous section is unique up to scalar multiples. As mentioned in $\S 1$, this will mean that a grid $\mathbf{k} \times \mathbf{n}$ for fixed $k$ and "large" $n$ has, in a sense, only one maximal antichain with the minimum splitting number. We will need the following standard results from matrix theory.

Lemma 3 (a) For all matrices $A, B$ of the same size, $\operatorname{rank}(A+B) \leq \operatorname{rank}(A)+\operatorname{rank}(B)$.

(b) Consider a square block-matrix $\left[\begin{array}{ll}A & B \\ C & D\end{array}\right]$, where $A$ and $D$ are square and $A$ is nonsingular. Then

$$
\operatorname{det}\left[\begin{array}{ll}
A & B \\
C & D
\end{array}\right]=\operatorname{det}\left(D-C A^{-1} B\right) \operatorname{det}(A)
$$

For example, see [4], Proposition 2 page 96 for (a) and Exercise 15 page 46 for (b).

Suppose that $A$ is a $k$-element antichain in $L=\mathbf{k} \times \mathbf{n}$, where $k$ is odd, and that $\bar{n}$ is its associated $(k+1)$-vector. Suppose also that for all $2^{k}$ orientations of $A$, at most $s_{k}|L|=s_{k} k n$ elements of $L$ are captured by each orientation. From $\S 2$ the number of elements of $L$ captured by any orientation is at least $\bar{v}_{o} \cdot \bar{n}$, and so $\bar{v}_{o} \cdot \bar{n} \leq s_{k} k n$ for all orientations of $A$. From (1) we have constants $\lambda_{i}>0$ so that

$$
\sum_{i=1}^{k+1} \lambda_{i}\left(\bar{v}_{o_{i}} \cdot \bar{n}\right)=s_{k} k n \sum_{i=1}^{k+1} \lambda_{i}
$$

for all orientations $o_{i}$ in the family $\mathcal{O}$ defined in Section 3. It follows from the last two sentences that $\bar{v}_{o_{i}} \cdot \bar{n}=s_{k} k n$ for all $o_{i} \in \mathcal{O}$. Thus

$$
M_{k} \bar{n}=s_{k} k J \bar{n}
$$

for the capture matrix $M_{k}$ of $\mathcal{O}$ defined in $\S 3$, and where now we consider $\bar{n}$ to be a column vector.

We claim that $\bar{n}$ is a scalar multiple of the vector $\bar{n}_{k}$ defined in Section 4 , and so the corresponding antichain $A$ defined there is the only antichain in $L$ whose splitting number is $s_{k}$. In other words,

Theorem 2 For any odd integer $k$, the vector $\bar{n}_{k}$ is, up to scalar multiples, the unique solution of $M_{k} \bar{x}=s_{k} k J \bar{x}$.

Proof. First we check that $\bar{n}_{k}$ is a solution of $M_{k} \bar{x}=s_{k} k J \bar{x}$. We know that (8) holds for $\bar{n}=\bar{n}_{k}$. We also know from $\S 4$ that $n=\sum \bar{n}_{k}=y_{k}$ and that for all $i$

$$
\bar{v}_{o_{i}} \cdot \bar{n}_{k} \leq s_{k} k y_{k}
$$

by (5). So as above, we have equality in (9) for all $i$, and $M_{k} \bar{n}_{k}=s_{k} k J \bar{n}_{k}$ by the definition of $M_{k}$. 
We wish to show that for odd integers $k, \operatorname{rank}\left(M_{k}-s_{k} k J\right)=k$. Since from $\S 3$

$$
M_{k}-s_{k} k J=C_{k}+\left((k-1)-s_{k} k\right) J=C_{k}-\frac{1}{y_{k}} J
$$

and $\operatorname{rank}(c J)=1$ for any nonzero scalar $c, \operatorname{rank}\left(M_{k}-s_{k} k J\right)$ is within 1 of $\operatorname{rank}\left(C_{k}\right)$ by Lemma 3(a). Thus, Theorem 2 will follow once we prove that $\operatorname{det}\left(C_{k}\right)$ is nonzero.

To do this, we prove in fact that $\operatorname{det}\left(C_{k}\right)$ is an odd integer! Simple computations show that

$$
\operatorname{det}\left(C_{1}\right)=\left|\begin{array}{ll}
0 & 1 \\
1 & 0
\end{array}\right|=-1 \quad \text { and } \quad \operatorname{det}\left(C_{3}\right)=\left|\begin{array}{cccc}
0 & 1 & -1 & 1 \\
-1 & 0 & 1 & 0 \\
0 & 1 & 0 & -1 \\
1 & -1 & 1 & 0
\end{array}\right|=3
$$

so the claim holds for $k=1$ and 3. Proceed by induction on $k$.

The matrices $C_{k}$, displayed in Section 3, have an obvious recursive structure, once a few rows and columns are moved. Notice that if rows 2 and $k$ and columns $(k+1) / 2$ and $(k+3) / 2$ are deleted from $C_{k}$, what we get is just $C_{k-2}$, in both of the cases $k \equiv 1 \bmod 4$ and $k \equiv 3 \bmod 4$. This prompts us to define the matrix $C_{k}^{\prime}$ from $C_{k}$ by moving rows 2 and $k$ to rows $k$ and $k+1$ [respectively] and moving columns $(k+1) / 2$ and $(k+3) / 2$ to columns $k$ and $k+1$ [respectively]. Then

$$
C_{k}^{\prime}=\left(\begin{array}{cc}
C_{k-2} & B \\
C & D
\end{array}\right)
$$

where $B$ is $(k-1) \times 2, C$ is $2 \times(k-1)$ and $D$ is $2 \times 2$. In fact, for $k \equiv 1 \bmod 4$,

$$
\begin{aligned}
B^{t} & =\left(\begin{array}{ccccccccc}
-1 & 1 & -1 & 1 & \ldots & -1 & 1 & -1 & 1 \\
1 & -1 & 1 & -1 & \ldots & 1 & -1 & 1 & -1
\end{array}\right), \quad D=\left(\begin{array}{cc}
0 & 1 \\
1 & 0
\end{array}\right), \quad \text { and } \\
C & =\left(\begin{array}{cccccccccccccccc}
1 & -1 & 1 & -1 & \cdots & 1 & -1 & 1 & -2 & -1 & 1 & -1 & \cdots & 1 & -1 & 1 \\
1 & -1 & 1 & -1 & \cdots & 1 & -1 & 1 & -1 & -2 & 1 & -1 & \cdots & 1 & -1 & 1
\end{array}\right),
\end{aligned}
$$

For $k \equiv 3 \bmod 4$, the results are similar: $D$ is again $\left(\begin{array}{ll}0 & 1 \\ 1 & 0\end{array}\right)$,

$$
B^{t}=\left(\begin{array}{ccccccccccccc}
1 & 1 & -1 & 1 & -1 & 1 & \ldots & -1 & 1 & -1 & 1 & -1 & -1 \\
-1 & -1 & 1 & -1 & 1 & -1 & \ldots & 1 & -1 & 1 & -1 & 1 & 1
\end{array}\right)
$$

and (for $k>3$ )

$$
C=\left(\begin{array}{llllllllllllllllll}
0 & 1 & -1 & 1 & -1 & \cdots & 1 & -1 & 1 & -2 & -1 & 1 & -1 & \cdots & 1 & -1 & 1 & 0 \\
0 & 1 & -1 & 1 & -1 & \cdots & 1 & -1 & 1 & -1 & -2 & 1 & -1 & \cdots & 1 & -1 & 1 & 0
\end{array}\right) .
$$

The number of consecutive transpositions needed to move row 2 of $C_{k}$ to the bottom of the matrix is $k-1$, an even number, and two more transpositions are then needed 
to move (original) row $k$ to the bottom. The number of transpositions needed to move column $(k+1) / 2$ to the right edge of $C_{k}$ is the same as the number subsequently required to move column $(k+3) / 2$ to the right edge. Thus an even number of row or column transpositions are required to change $C_{k}$ into $C_{k}^{\prime}$, so $\operatorname{det}\left(C^{\prime}{ }_{k}\right)=\operatorname{det}\left(C_{k}\right)$.

Suppose $k \geq 5$ and that $\operatorname{det}\left(C_{k-2}\right)$ is an odd integer. Using Lemma 3(b) and noting that $C_{k-2}$ is invertible from the induction hypothesis, we have

$$
\operatorname{det}\left(C_{k}\right)=\operatorname{det}\left(C_{k}^{\prime}\right)=\operatorname{det}\left(C_{k-2}\right) \operatorname{det}(S)
$$

where $S=D-C C_{k-2}^{-1} B$. To simplify notation, let $A=C_{k-2}$, and

$$
C A^{-1}=\left(\begin{array}{llll}
a_{1} & a_{2} & \ldots & a_{k-1} \\
b_{1} & b_{2} & \ldots & b_{k-1}
\end{array}\right)
$$

Then for $j=1,2, \ldots, k-1$,

$$
\begin{aligned}
b_{j}-a_{j} & =\operatorname{row}_{2}(C) \cdot \operatorname{col}_{j}\left(A^{-1}\right)-\operatorname{row}_{1}(C) \cdot \operatorname{col}_{j}\left(A^{-1}\right) \\
& =\left[\operatorname{row}_{2}(C)-\operatorname{row}_{1}(C)\right] \cdot \operatorname{col}_{j}\left(A^{-1}\right) \\
& =\left(0 \ldots 0 \quad \frac{k-1}{2}\right. \\
& =\left(A^{-1}\right)_{\frac{k-1}{2}, j}-\left(A^{-1}\right)_{\frac{k+1}{2}, j} \\
& =\alpha_{j}, \quad \text { say, }
\end{aligned}
$$

so each $b_{j}=a_{j}+\alpha_{j}$.

For odd indices $k$, it is immediate from the definition of the matrices $C_{k}$ that $\operatorname{row}_{k-i}(A)$ is the reverse of $\operatorname{row}_{i}(A)$ (or, more picturesquely, that $A$ is unchanged if rotated $180^{\circ}$ ). It follows by symmetry that $A^{-1}$ must have the same property. Thus

$$
\begin{aligned}
\alpha_{j} & =\left(A^{-1}\right)_{\frac{k-1}{2}, j}-\left(A^{-1}\right)_{\frac{k+1}{2}, j} \\
& =\left(A^{-1}\right)_{\frac{k+1}{2}, k-j}-\left(A^{-1}\right)_{\frac{k-1}{2}, k-j} \\
& =-\alpha_{k-j} .
\end{aligned}
$$

Therefore, $C A^{-1}=$

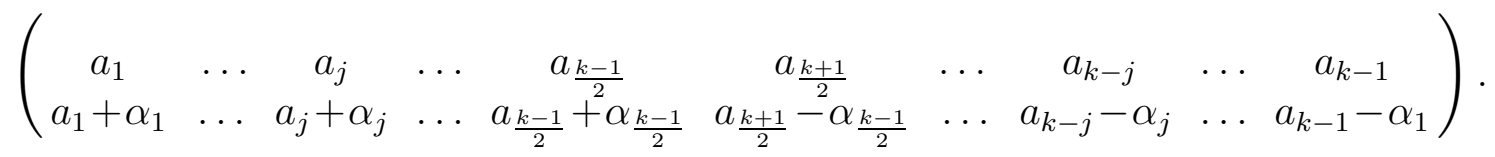

Now we give the complete argument that $\operatorname{det}\left(C_{k}\right)$ is an odd integer in case $k \equiv 1 \bmod 4$ - the other case is almost identical. We wish to find $C A^{-1} B$, which is a $2 \times 2$ matrix. If we let

$$
a=\sum_{i=1}^{k-1}(-1)^{i} a_{i} \quad \text { and } \quad \alpha=2\left(\sum_{i=1}^{(k-1) / 2}(-1)^{i} \alpha_{i}\right)
$$


then

$$
C A^{-1} B=\left(\begin{array}{cc}
a & -a \\
a+\alpha & -(a+\alpha)
\end{array}\right)
$$

and

$$
S=\left(\begin{array}{ll}
0 & 1 \\
1 & 0
\end{array}\right)-\left(\begin{array}{cc}
a & -a \\
a+\alpha & -(a+\alpha)
\end{array}\right)=\left(\begin{array}{cc}
-a & 1+a \\
1-(a+\alpha) & a+\alpha
\end{array}\right) .
$$

Therefore

$$
\operatorname{det}(S)=-a(a+\alpha)-(1+a)+(1+a)(a+\alpha)=\alpha-1 .
$$

We need to take a closer look at $\alpha_{j}$ to show that $\operatorname{det}\left(C_{k}\right)$ is an odd integer. For each $1 \leq j \leq(k-1) / 2$,

$$
\alpha_{j}=\left(A^{-1}\right)_{\frac{k-1}{2}, j}-\left(A^{-1}\right)_{\frac{k+1}{2}, j}=\frac{1}{\operatorname{det}(A)}\left[(\operatorname{adj}(A))_{\frac{k-1}{2}, j}-(\operatorname{adj}(A))_{\frac{k+1}{2}, j}\right] .
$$

[We are using standard notation: $\operatorname{adj}(A)$ is the classical adjoint of $A$.] Observe that

$$
r_{j}=(\operatorname{adj}(A))_{\frac{k-1}{2}, j}-(\operatorname{adj}(A))_{\frac{k+1}{2}, j}
$$

is an integer since $A$ and thus $\operatorname{adj}(A)$ is an integer matrix, so $r=\sum_{i=1}^{(k-1) / 2}(-1)^{i} r_{i}$ is an integer. Now we have

$$
\alpha=2\left(\sum_{i=1}^{(k-1) / 2}(-1)^{i} \alpha_{i}\right)=\frac{2}{\operatorname{det}(A)}\left(\sum_{i=1}^{(k-1) / 2}(-1)^{i} r_{i}\right)=\frac{2 r}{\operatorname{det}(A)} .
$$

Apply the induction hypothesis: $\operatorname{det}(A)=2 s-1$ for some integer $s$, so

$$
\begin{aligned}
\operatorname{det}\left(C_{k}\right) & =\operatorname{det}(A) \operatorname{det}(S) \\
& =(2 s-1)(\alpha-1) \\
& =(2 s-1)\left(\frac{2 r}{2 s-1}-1\right) \\
& =2 r-(2 s-1) \\
& =2(r-s)+1,
\end{aligned}
$$

an odd integer. This completes the proof of Theorem 2.

Incidentally, from the above proof we have

$$
\left(M_{k}-s_{k} k J\right) \bar{n}_{k}=\left(C_{k}-\frac{1}{y_{k}} J\right) \bar{n}_{k}=C_{k} \bar{n}_{k}-\overline{1},
$$

so the property $M_{k} \bar{n}_{k}=s_{k} k J \bar{n}_{k}$ is equivalent to

$$
C_{k} \bar{n}_{k}=\overline{1}
$$

Compare this to the property $C_{k}^{t} \bar{\lambda}=\overline{1}$ which we derived in $\S 3$. 


\section{Nonuniqueness of $\bar{n}_{k}$ for $k$ even; the set of capture vectors; open problems}

In $\S 5$ we proved that, for odd $k$, the vector $\bar{n}_{k}$ of $\S 4$ is, up to scalar multiples, the unique vector satisfying $M_{k} \bar{x}=s_{k} k J \bar{x}$ and so it determines the unique (up to proportions) antichain in $\mathbf{k} \times \mathbf{n}$ with minimal splitting number. However, this is not true for all even $k$. One difference is that the matrices $C_{k}$ do not appear to be invertible for even $k$.

We look at the case $k=4$ as an example. Here, the splitting number is $s_{4}=7 / 9$, and any vector $\bar{x}=(2-t, 4-2 t, 3,2 t, t)$, where $3 / 5 \leq t \leq 7 / 5$, turns out to satisfy $\bar{v}_{o} \cdot \bar{x} \leq s_{4}\left(4 y_{4}\right)=4(7 / 9) 9=28$ for all $2^{4}$ orientations $o$ of $A$. (Since $\sum \bar{x}=9=y_{4}$, this is (5) for $k=4$ and with $\bar{n}_{4}$ replaced by $\bar{x}$.) Therefore scalar multiples of each such vector will yield, in the corresponding grid, a maximal antichain which can capture at most $7 / 9$ of the grid in the limit.

To show this we can mimic the methods of $\S 4$, although the lack of symmetry in $\bar{x}$ means we have more work to do. To establish the counterpart of (7) when $k=4$, we need to prove that $\bar{v}_{a} \cdot \bar{x}=28$ for each of the two alternating orientations $a$. For $a=a_{\uparrow}$ we get

$$
\bar{v}_{a_{\uparrow}} \cdot \bar{x}=[\overline{3}+(0,1,-1,1,0)] \cdot(2-t, 4-2 t, 3,2 t, t)=9 \cdot 3+1=28,
$$

and similarly for $a=a_{\downarrow}$ we get

$$
\bar{v}_{a_{\downarrow}} \cdot \bar{x}=[\overline{3}+(1,-1,1,-1,1)] \cdot(2-t, 4-2 t, 3,2 t, t)=9 \cdot 3+1=28 .
$$

To prove that $\bar{v}_{o} \cdot \bar{x} \leq 28$ for all orientations $o$, we could just do the calculation for each of the remaining 14 orientations separately. However for the most part we can use the same idea as in $\S 4$. That is, we define, for every non-alternating orientation $o$, another orientation $\hat{o}$ which is "closer" to being alternating, and prove the counterpart of (6), namely that

$$
\left(\bar{v}_{\hat{o}}-\bar{v}_{o}\right) \cdot \bar{x} \geq 0 .
$$

Again we will write $\bar{v}_{o}=\left(a_{1}, \ldots, a_{5}\right)$ and $\bar{v}_{\hat{o}}=\left(b_{1}, \ldots, b_{5}\right)$. We consider the orientations in groups.

Case (i): $o=\uparrow \uparrow \uparrow \ddagger$ (where each of $\dagger$ and $\ddagger$ will mean either $\uparrow$ or $\downarrow$, so this case handles four orientations at once). Then we let $\hat{o}=\downarrow \uparrow \uparrow \ddagger$, where only the first symbol has been changed. It is easy to check that $b_{1} \geq a_{1}+2, b_{2}=a_{2}-1$, and $b_{i}=a_{i}$ for $i \geq 3$, so

$$
\left(\bar{v}_{\hat{o}}-\bar{v}_{o}\right) \cdot \bar{x} \geq(2,-1,0,0,0) \cdot(2-t, 4-2 t, 3,2 t, t)=0 .
$$

Case (ii): $o=\downarrow \downarrow \dagger \ddagger$. Then we similarly let $\hat{o}=\uparrow \downarrow \dagger \ddagger$, and we get $b_{1}=a_{1}-1, b_{2}=a_{2}+1$, $b_{3}=a_{3}+1$, and $b_{i} \geq a_{i}$ for $i \geq 4$. Thus

$$
\left(\bar{v}_{\hat{o}}-\bar{v}_{o}\right) \cdot \bar{x} \geq(-1,1,1,0,0) \cdot(2-t, 4-2 t, 3,2 t, t)=5-t \geq 0 .
$$

Case (iii): $o=\downarrow \uparrow \uparrow \ddagger$. Let $\hat{o}=\uparrow \downarrow \uparrow \ddagger$, for which $b_{1}=a_{1}-1, b_{2} \geq a_{2}+3, b_{3}=a_{3}-1$, and $b_{i} \geq a_{i}$ for $i \geq 4$. Thus

$$
\left(\bar{v}_{\hat{o}}-\bar{v}_{o}\right) \cdot \bar{x} \geq(-1,3,-1,0,0) \cdot(2-t, 4-2 t, 3,2 t, t)=7-5 t \geq 0,
$$


since $t \leq 7 / 5$.

Case (iv): $o=\uparrow \downarrow \downarrow \ddagger$. Let $\hat{o}=\downarrow \uparrow \downarrow \ddagger$, and we get $b_{1}=a_{1}+1, b_{2}=a_{2}-2, b_{3}=a_{3}+1$, $b_{4}=a_{4}+1$, and $b_{5} \geq a_{5}$. Thus

$$
\left(\bar{v}_{\hat{o}}-\bar{v}_{o}\right) \cdot \bar{x} \geq(1,-2,1,1,0) \cdot(2-t, 4-2 t, 3,2 t, t)=5 t-3 \geq 0,
$$

since $t \geq 3 / 5$.

This leaves two remaining orientations, which we handle directly:

Case (v): For $o=\uparrow \downarrow \uparrow \uparrow$, we have

$$
\bar{v}_{o} \cdot \bar{x}=(3,4,1,3,4) \cdot(2-t, 4-2 t, 3,2 t, t)=25-t \leq 28 .
$$

Case (vi): For $o=\downarrow \uparrow \downarrow \downarrow$, we have

$$
\bar{v}_{o} \cdot \bar{x}=(4,2,4,3,2) \cdot(2-t, 4-2 t, 3,2 t, t)=28 .
$$

This shows that $\bar{x}=(2-t, 4-2 t, 3,2 t, t)$ for $3 / 5 \leq t \leq 7 / 5$ always defines a maximal antichain with minimum splitting number. If we set $t=1$, then we get the symmetric vector $\bar{n}_{4}=(1,2,3,2,1)$ given in $\S 4$ and included as row 5 of the triangular array at the end of $\S 4$. But other values of $t$ give nonsymmetric solutions.

We did not consider the case $k=2$ in this paper, as the matrix $M_{k}$ is not defined for $k=2$. But the value $s_{2}=2 / 3$ was derived in [3], and the reader can easily check that, for any vector $(t, 1,2-t)$ where $1 / 2 \leq t \leq 3 / 2$, at most $2 / 3$ of the $\mathbf{2} \times \mathbf{n}$ grid is captured by any of the four orientations of the corresponding two-element maximal antichain. When $t=1$ we get the symmetric vector $(1,1,1)$ which makes up the third row of the triangular array at the end of $\S 4$. However, other permissible values of $t$ give nonsymmetric vectors (and thus antichains) with the same splitting number.

Problem 1. For $k$ even, is $\bar{n}_{k}$ the only vector $\bar{x}$ (up to scalar multiples) which is symmetric (that is, $\bar{x}^{r}=\bar{x}$ ) and satisfies $M_{k} \bar{x}=s_{k} k J \bar{x}$ ?

In this paper we have determined the number $s_{k}\left(\mathcal{G}_{k}\right)$, which tells us how much of the $\mathbf{k} \times \mathbf{n}$ grid can be captured by splitting a $k$-element maximal antichain. It seems to us, however, that other maximal antichains of $\mathbf{k} \times \mathbf{n}$, which must necessarily contain fewer elements, should split so as to capture even more elements, for instance since they are "further away" from the middle of the lattice. We have not succeeded in proving this, so the following is a second open question:

Problem 2. Is $s\left(\mathcal{G}_{k}\right)=s_{k}\left(\mathcal{G}_{k}\right)$ for all $k$ ?

Finally, let's look briefly at what we could call the full set of capture vectors for each positive integer $k$. For each of the $2^{k}$ orientations $o$ of an antichain in $G_{k}=\mathbf{k} \times \mathbf{n}$, there is an associated $(k+1)$-element capture vector $\bar{v}_{o}$. Let $\mathcal{O}_{k}$ denote the set of all orientations, and let $V_{k}$ denote the set of $2^{k}(k+1)$-element capture vectors.

While it is easy to generate the $2^{k}$ vectors in $V_{k}$ recursively, we do not have a pleasing characterization of these integer vectors. We do have a couple of observations about this 
family of vectors that are worth recording, particularly as they lead to some interesting open problems.

As was noted at the beginning of $\S 2$, any member $o$ of $\mathcal{O}_{k}$ can be regarded as a $k$ sequence of $\uparrow$ 's and $\downarrow$ 's. Identify $\uparrow$ with 0 and $\downarrow$ with 1 , so $\mathcal{O}_{k}$ is the set of all binary sequences of length $k$. Let $\prec$ denote the usual lexicographic ordering of $\mathcal{O}_{k}$. Let's also take $\prec$ to be the usual lexicographic ordering on the integer vectors in $V_{k}$. We claim the following:

$$
o^{\prime} \prec o \text { in } \mathcal{O}_{k} \quad \text { if and only if } \quad \bar{v}_{o^{\prime}} \prec \bar{v}_{o} \text { in } V_{k} \text {. }
$$

We should say that an orientation is easily recovered from its capture vector: if $\bar{v}_{o}=$ $\left(a_{1}, \ldots, a_{k+1}\right)$ then the $i^{\text {th }}$ arrow in $o$ is $\uparrow$ if and only if $a_{i}>a_{i+1}$. To establish (10), let's consider the value $a_{i}$. Note that $a_{i}=a_{i}^{u}+a_{i}^{d}$ where $a_{i}^{u}$ is the position of the closest $\uparrow$ to the left of, or at, position $i-1$ and $a_{i}^{d}$ is $k+1$ minus the position of the closest $\downarrow$ to the right of, or at, position $i$. Let $\bar{v}_{o^{\prime}}=\left(b_{1}, \ldots, b_{k+1}\right)$ and suppose that $o^{\prime} \prec o$. Suppose that the first difference occurs in position $i$ : thus, $o^{\prime}$ has $\uparrow$ in position $i$ and $o$ has $\downarrow$. Then $b_{i}^{u}=a_{i}^{u}$, since the sequences $o$ and $o^{\prime}$ are identical to the left of position $i$, and $a_{i}^{d}=k+1-i>b_{i}^{d}$, so $b_{i}<a_{i}$. It is easy to see that for $j<i, b_{j} \leq a_{j}$, establishing (10).

The componentwise order on vectors is likely the most familiar. Using $\leq$ to denote this, $\mathcal{O}_{k}$ under $\leq$ is just the usual Boolean $k$-cube. On the other hand, we assert that

$$
V_{k} \text {, under } \leq \text {, is an antichain. }
$$

To prove (11), let $o$ and $o^{\prime}$ be exactly as in the verification of (10). In particular, $o \prec o^{\prime}$ and $b_{i}<a_{i}$. Consider $a_{i+1}$ and $b_{i+1}$. Then $b_{i+1}^{u}=i$ and $a_{i+1}^{u}<i$, so either $b_{i+1}>a_{i+1}$, or $b_{i+1}^{d}<a_{i+1}^{d}$, that is, the nearest $\downarrow$ to the right of position $i$ is closer in $o$ than in $o^{\prime}$. If the latter, say this $\downarrow$ in $o$ is in position $r$. If $r=k$ then $o^{\prime}$ has $\uparrow$ in position $k$ and $o$ does not, meaning $b_{k+1}=k+1>a_{k+1}$. If $r<k, b_{r+1}^{u}=r$ and $a_{r+1}^{u}<r$, so we can replace $i$ by $r$ and continue. In some coordinate $\bar{v}_{o^{\prime}}$ is greater than $\bar{v}_{o}$, proving (11).

We know (see the remark at the end of $\S 4$ ) that given any capture vector $\bar{v}_{o}$ for $o$ in either of the families $\mathcal{O}$ defined in $\S 3$, and vector $\bar{n}_{k}$ as defined in $\S 4$,

$$
\bar{v}_{o} \cdot \bar{n}_{k}=s_{k}(k n) \geq \bar{v} \cdot \bar{n}_{k}
$$

for all $\bar{v} \in V_{k}$. We wonder if this is true more generally, particularly as (11) does not militate against it.

Problem 3. Given any capture vector $\bar{v}$ in $V_{k}$, will there always exist a vector $\bar{n}$ of positive integers so that $\bar{v} \cdot \bar{n} \geq \bar{w} \cdot \bar{n}$ for all capture vectors $\bar{w} \in V_{k}, \bar{w} \neq \bar{v}$ ?

If true, this would say that for every orientation $o$ there is some maximal antichain (as defined by the vector $\bar{n}$ ) of some grid $\mathbf{k} \times \mathbf{n}$ for which $o$ captures at least as much of the grid as any other orientation.

Problem 4. Given any capture vector $\bar{v}$ in $V_{k}$, can there ever exist nonnegative real numbers $\mu_{1}, \ldots, \mu_{2^{k}-1}$ satisfying $\sum_{i=1}^{2^{k}-1} \mu_{i}=1$ so that $\bar{v} \leq \sum_{i=1}^{2^{k}-1} \mu_{i} \bar{w}_{i}$, where $\left\{\bar{w}_{1}, \ldots, \bar{w}_{2^{k-1}}\right\}$ is the set of all vectors in $V_{k}$ not equal to $\bar{v}$ ? 
This asks whether any capture vector can be dominated by a convex combination of the other capture vectors. A negative answer would seem a considerable strengthening of the observation in (11); for $k \leq 4$, we have obtained a negative answer.

Actually, these last two problems may be related. The following may turn out to be a somewhat reckless combination of both.

Problem 5. For arbitrary vectors $\bar{v}, \bar{v}_{1}, \bar{v}_{2}, \ldots, \bar{v}_{k}$ in $\mathbb{R}^{n}$, is it always true that either (i) there are nonnegative real numbers $c_{i}, 1 \leq i \leq k$, such that

$$
\sum_{i=1}^{k} c_{i}=1 \text { and } \bar{v} \leq \sum_{i=1}^{k} c_{i} \bar{v}_{i}
$$

or

(ii) there exists a nonnegative nonzero vector $\bar{u} \in \mathbb{R}^{n}$ such that $\bar{v} \cdot \bar{u} \geq \bar{v}_{i} \cdot \bar{u}$ for all $i$ ? This is easily proved for $k=1$ and for $n=1$ and we have a proof in the case $k=2$.

\section{References}

[1] R. Ahlswede, P. L. Erdős, and N. Graham, A splitting property of maximal antichains, Combinatorica 15 (1995), 475 - 480.

[2] D. Duffus and B. Sands, Minimum sized fibres in distributive lattices, J. Australian Math. Soc. 70 (2001), 337 - 350.

[3] D. Duffus and B. Sands, Finite distributive lattices and the splitting property, Algebra Universalis, in press.

[4] P. Lancaster and M. Tismenetsky, The Theory of Matrices, Second Edition, Academic Press, 1985.

[5] http://mathworld.wolfram.com/PellNumber.html 